1 Main Document with Figures and Tables

2

\title{
Integrative analysis of the salt stress response in cyanobacteria
}

4

5 Stephan Klähn ${ }^{1,2 \star}$, Stefan Mikkat ${ }^{3 \star}$, Matthias Riediger², Jens Georg ${ }^{2}$, Wolfgang R. Hess ${ }^{2}$,

6 Martin Hagemann $n^{4,5, A}$

71 - Helmholtz-Centre for Environmental Research - UFZ, Department of Solar Materials,

8 Leipzig, Germany

92 - University of Freiburg, Faculty of Biology, Genetics and Experimental Bioinformatics, 10 Freiburg, Germany

113 - Core Facility Proteome Analysis, Rostock University Medical Center, Rostock, Germany

124 - University of Rostock, Institute of Biosciences, Dept. Plant Physiology, Rostock, Germany

135 - Department Life, Light \& Matter, University of Rostock, Rostock, Germany

* The first two authors contributed equally to this study.

A - corresponding author: Universität Rostock, Institut für Biowissenschaften, Abt. Pflanzenphysiologie, A.-Einstein-Str. 3, D-18059 Rostock, Germany, Tel. +49(0)3814986110, Fax.+49(0)3814986112, Email. martin.hagemann@uni-rostock.de

Running title: Salt acclimation of cyanobacteria

SK: stephan.klaehn@ufz.de; orcid: 0000-0002-2933-486X

SM: stefan.mikkat@med.uni-rostock.de

MR: matthias.riediger@biologie.uni-freiburg.de

JG: jens.georg@biologie.uni-freiburg.de; orcid: 0000-0002-7746-5522

WRH: wolfgang.hess@biologie.uni-freiburg.de; orcid: 0000-0002-5340-3423 


\section{Abstract}

Microorganisms evolved specific acclimation strategies to thrive in environments of high or fluctuating salinities. Here, salt acclimation in the model cyanobacterium Synechocystis sp. PCC 6803 was analyzed by integrating transcriptomic, proteomic and metabolomic data. A dynamic reorganization of the transcriptome and proteome occurred during the first hours after salt shock, e.g. involving the upregulation of genes to activate compatible solute biochemistry balancing osmotic pressure. The massive accumulation of glucosylglycerol then had a measurable impact on the overall carbon and nitrogen metabolism. In addition, we observed the coordinated induction of putative regulatory RNAs and of several proteins known for their involvement in other stress responses. Overall, salt-induced changes in the proteome and transcriptome showed good correlations, especially among the stably upregulated proteins and their transcripts. We define an extended salt stimulon comprising proteins directly or indirectly related to compatible solute metabolism, ion and water movements, and a distinct set of regulatory RNAs involved in post-transcriptional regulation. Our comprehensive data set provides the basis for engineering cyanobacterial salt tolerance and to further understand its regulation.

\section{Key words}

compatible solute/ ion transport/ regulatory RNAs/ transcriptome-proteome correlation/ salinity stress response

\section{Introduction}

Salinity is a prominent environmental factor determining the natural distribution of microorganisms, as approximately $97 \%$ of the water resources contain more than $30 \mathrm{~g}$ salt (mainly sodium chloride) per liter. Accordingly, the capability of microorganisms to cope with high or changing salinities is crucial, not only in aquatic environments but also in terrestrial habitats, in which the alternation between evaporation and rainfall can rapidly change salt concentrations. In a hypersaline environment, in which the external salt concentration exceeds the cellular ion content, microorganisms have to manage two major challenges: (i) the low external water potential results in water loss from the cell and collapse of turgor pressure, and (ii) inorganic ions permeate into cells along the electrochemical gradient, which could compromise the structure of critical macromolecules. Accordingly, most microorganisms feature acclimation strategies aiming at maintaining a high water and low inorganic ion content in the cell. This so-called "salt-out" strategy is based on the active extrusion of inorganic ions accompanied by the accumulation of compatible solutes, i.e. 
highly soluble, non-toxic, low molecular-mass organic compounds, for osmotic equilibrium (e.g., Hagemann, 2011).

Cyanobacteria are a morphologically and physiologically diverse group of photoautotrophic bacteria that are found in nearly all light-exposed habitats including environments with different salinities such as freshwaters, oceans, and hypersaline ponds or soil surfaces in temperate and arid climates (Whitton and Potts, 2000). Previous studies of cyanobacterial salt tolerance revealed that those with low salt tolerance, mainly freshwater and soil cyanobacteria, accumulate the sugars sucrose and/or trehalose, those with moderate tolerance (mainly marine strains) synthesize the heteroside glucosylglycerol (GG), whereas halophilic strains (found in hypersaline habitats) usually contain glycine betaine (Reed et al., 1986; Hagemann, 2011). Some deviations from these preferences have been documented as well (e.g., Klähn et al., 2010a; Pade et al., 2012, 2016).

Cyanobacterial salt acclimation has been investigated in great detail using the model strain Synechocystis sp. PCC 6803 (Synechocystis 6803). This unicellular strain was originally isolated from a freshwater pond (Stanier et al., 1971). Nevertheless, Synechocystis 6803 represents a truly euryhaline organism able to grow in freshwater but also in media containing salt concentrations twice as high as in seawater (Reed and Stewart, 1985). The early availability of both, genetic tools (Grigorieva and Shestakov, 1982) and the complete genome sequence (Kaneko et al., 1996) established Synechocystis 6803 as photoautotrophic model organism. Using this strain, the molecular basis of salt-induced GG synthesis has been characterized, which is performed by the enzymes GG-phosphate synthase and GG-phosphate phosphatase, encoded by the genes ggpS (sll1566) and ggpP (stpA, slr0746), respectively (Hagemann et al., 1997; Marin et al., 1998). Today, Synechocystis 6803 is the best investigated photoautotrophic prokaryote represented by more than 3700 scientific publications, and has become popular as chassis for the introduction of pathways for the photosynthetic production of biofuels or chemical feedstock (e.g., Hagemann and Hess, 2018; Liu et al., 2019a). Salt-tolerant cyanobacterial strains such as Synechocystis 6803 also permit large scale cultivations in saline waters making the process more sustainable by avoiding competition for limited freshwater resources (Chisti, 2013).

Over the past two decades, omics technologies have been applied to study salt acclimation of cyanobacteria, particularly Synechocystis 6803. Genome-wide transcriptome analyses revealed the differential expression of hundreds of genes after sudden increases in the external salinity and allowed the identification of several potential regulatory proteins involved in their stress-induced expression (Kanesaki et al., 2002; Marin et al., 2003; Shoumskaya et al., 2005). However, most of these genes were only transiently induced or repressed. In long- 
term salt-acclimated cells, the expression of only 39 genes remained significantly enhanced (Marin et al., 2004). Later on, 2D-gel-based proteomics displayed a snapshot of the saltregulated proteome, which identified 45 stably salt-induced soluble proteins (Fulda et al., 2006), while 20 proteins of the membrane fraction appeared to be salt-regulated (Huang et al., 2006). In the meantime, advanced omics technologies such as different RNA-seq technologies were established, which, for example, revealed that also large numbers of nonprotein-coding RNAs (ncRNAs) are transcribed in Synechocystis 6803 (Mitschke et al., 2011; Kopf et al., 2014; Billis et al., 2014). Among them, two classes can be differentiated, cisencoded antisense RNAs (asRNAs) transcribed from the opposite strand within proteincoding genes, and trans-encoded small regulatory RNAs (sRNAs) (Kopf and Hess, 2015; Georg and Hess, 2018). Some of the newly annotated asRNAs were demonstrated to act as regulators of their cognate mRNAs (Dühring et al., 2006; Sakurai et al., 2012) or sRNAs functioning in the acclimation response to changing environmental conditions (Georg et al., 2014, 2017; Klähn et al., 2015; Zhan et al., 2021). Moreover, tremendous progress has been made in the investigation of cyanobacteria using gel-free technologies for proteomics (Wegener et al., 2010; Gao et al., 2015a; Spät et al., 2021) or metabolomics (reviewed by Schwarz et al., 2013).

Here, we combined transcriptomic with proteomic and metabolomic approaches for a comprehensive characterization of the salt acclimation process in Synechocystis 6803. Previous studies (e.g., Marin et al., 2004; Fulda et al., 2006) showed that salt acclimation is a highly dynamic process, in which many genes/proteins showed an early but mostly transient response before long-term salt acclimation leads to stable, physiological meaningful changes in the gene/protein expression pattern. Therefore, we sampled Synechocystis 6803 cells at different time points up to 7 days after transfer from $\mathrm{NaCl}$-free into medium containing 684 $\mathrm{mM} \mathrm{NaCl}$ (equal to $4 \% \mathrm{NaCl}$ ). In addition to many salt-regulated proteins and their corresponding mRNAs, we identified several potentially regulatory asRNAs and sRNAs to be salt-stimulated as well. Finally, metabolomics revealed that the massive accumulation of the compatible solute GG has a broader impact on the overall primary carbon and nitrogen metabolism.

\section{Results}

The response of Synechocystis 6803 to $\mathrm{NaCl}$-induced hyperosmotic conditions (salt stress) was analyzed on transcriptome, proteome and metabolome levels at different time scales (Fig. 1). Changes in the expression profiles were first analyzed at a global scale. Then, selected examples were examined in a comprehensive way using all data sets. 


\section{Global transcriptome analysis}

In the present study a microarray platform was used to detect RNA levels directly, without reverse transcription. This microarray contained probes for all protein-coding genes also covering so far non-annotated open reading frames as well as for ncRNAs such as cis-acting asRNAs and trans-acting sRNAs previously identified in Synechocystis 6803 (Mitschke et al., 2011; Kopf et al., 2014). In total the microarray covered 3364 mRNAs, 1940 asRNAs and 602 sRNA candidates. Previous studies analyzing the salt transcriptome of Synechocystis 6803 used DNA microarrays only covering 3079 mRNAs and no ncRNAs (Marin et al., 2004). Here, the microarray was hybridized with total RNA extracted from control cells $(0 \% \mathrm{NaCl})$ and from cells exposed to $4 \%$ of $\mathrm{NaCl}$ for $0.5,2$, and $24 \mathrm{~h}$. The time points were chosen to permit comparison with previously published microarray data (Marin et al., 2004). Gene expression changes along the Synechocystis 6803 chromosome are shown in the Suppl. Genome Plots.

For the selection of differentially expressed genes, we applied typical cut-off criteria, i.e. $\log _{2}$ fold change $\geq|1|, p$-value $<0.05$. As the transcriptome composition was highly dynamic at the different time points, we first focused on protein coding genes (mRNAs). Compared to control conditions (i.e., cells grown in $\mathrm{NaCl}$-free BG11 medium), several hundred mRNAs showed a changed abundance 0.5 and $2 \mathrm{~h}$ after salt addition, while after $24 \mathrm{~h}$ only 87 were up- and 31 down-regulated (Table 1; volcano plots of transcriptional changes are displayed in Suppl. Fig. S1), consistent with the previous report by Marin et al. (2004). Only a few mRNAs were significantly changed at all sampling points, i.e. 31 showed elevated levels and 19 were down-regulated.

To evaluate the microarray data set systematically, a cluster analysis was performed using mfuzz (Kumar and Futschik, 2007). Initially, 8538 transcript types were differentiated, including all mRNAs but also 5'UTRs, asRNAs, sRNAs and transcripts derived from start sites within genes; Suppl. Table S2). Two clusters (cluster 1 and 2) include transcripts that were induced and two other clusters (cluster 3 and 4) include transcripts that were repressed at specific time points after salt addition (Fig. 2A). Transcripts in cluster 1 and 3 peaked at $0.5 \mathrm{~h}$, while transcripts in cluster 2 and 4 peaked at the $2 \mathrm{~h}$ time point. The majority of genes in all clusters, most pronounced in case of cluster 3 , showed a clear tendency to return to the initial values at the end of the time course indicating that the short-term acclimation was complete.

Specific transcript types showed an uneven distribution in the different clusters, i.e. clusters 1 and 2 contain a significantly higher number of asRNAs and lower number of sRNAs while cluster 3 and 4 showed the opposite. Protein-coding transcripts are more present in the downregulated clusters (780 mRNAs) than in the upregulated clusters (690 mRNAs) (Fig. 
2B). Functional enrichment analysis of the proteins encoded by mRNAs in each cluster was performed according to annotations from KEGG Orthology (KO) terms. On the one hand, mRNAs within the rapidly induced cluster 1 are enriched in proteins associated to replication and repair, cofactor biosynthesis, signaling, and transport, while the mRNAs of cluster 2 are enriched in protein families associated to transport, genetic information processing such as chaperones and folding catalysts, ribosome biogenesis, or transcription factors (Fig. 2C). On the other hand, transiently repressed mRNAs in the clusters 3 and 4 showed similar functionalities, and thus were analyzed jointly. The most pronounced functional enrichment could be seen for transcripts encoding proteins associated to energy and metabolism, such as oxidative phosphorylation, photosynthesis, nitrogen metabolism, or related metabolic functions, such as pathways of porphyrin and chlorophyll metabolism, lipid biosynthesis or pathways for carbon metabolism.

\section{2}

183

184

185

186

187

188

189

190

191

192

193

194

\section{Salt-regulated asRNA:mRNA pairs}

Most of the salt-induced changes of asRNA levels were transient, consistent with the observations for mRNAs. Our analysis considered only asRNAs that overlap on the opposite strand with the respective mRNAs, which led to the identification of 79 inversely regulated and 82 co-regulated asRNA/mRNA pairs (Fig. 3) at a Pearson correlation coefficient $\geq|0.65|$ (details in Table S4). Previous work in Synechocystis 6803 showed that both modes of regulation can be functionally relevant (Dühring et al., 2006; Eisenhut et al., 2012; Sakurai et al., 2012).

Among the asRNA/mRNA pairs is sll1862 (Fig. 3D) encoding one of the most abundant salt shock proteins (see below). The massive accumulation of stress protein SIl1862 is correlated by the inversely related levels of its mRNA versus asRNA. Another asRNA seems to be involved in controlling the salt-stimulated expression of s/r0082 (Fig. 3D), which encodes the ribosomal protein S12 methylthiotransferase RimO. This gene was early detected to be saltinduced by subtractive hybridization (Vinnemeier and Hagemann, 1999). It is transcribed in an operon together with the RNA helicase CrhR, which has been shown to be involved in multiple stress responses and is regulated by redox changes (Ritter et al., 2020). Moreover, the mRNA level of sll0923 encoding the tyrosine kinase Wzc was less abundant after salt addition (Wzc protein level was lowered with a FC of 0.53), while the corresponding asRNA showed increased abundance (Fig. 3C). WzC is involved in the synthesis of extracellular polysaccharides (EPS; Pereira et al., 2019), hence, the lowered expression of sll0923 is consistent with observation of reduced EPS synthesis in salt-acclimated Synechocystis 6803 cells (Kirsch et al., 2017). Among the 56 mRNA/asRNA pairs showing similar induction patterns, the gene s/r0953 encoding sucrose-phosphate phosphatase showed higher mRNA as well as asRNA levels (Fig. $3 \mathrm{E}$ ). It could be assumed that in this case the asRNA supports 
the stability of the s/r0953 mRNA contributing to the transiently elevated sucrose levels in salt-treated cells.

Moreover, we identified also not previously known salt-regulated transcripts, e.g. the sll1470 asRNA (Fig. 3). Although this asRNA originates from a TSS downstream of sll1470, it overlaps the 3' end of the gene and a clear anti-correlation with the transcript accumulation of sll1470 during salt shock was detected (Fig. 3C). Gene sll1470 encodes the large subunit of 3-isopropylmalate dehydratase, an important enzyme connecting pyruvate metabolism with leucine/isoleucine/valine biosynthesis. Therefore, its lowered expression during early salt shock likely contributes to the metabolic reorganization towards synthesizing GG consistent with the lowered valine accumulation (see below).

\section{Salt-regulated sRNAs}

217 The used microarray also permitted to search for salt-induced changes in the abundance of 218 sRNAs, which are in contrast to asRNAs encoded in trans. Interestingly, according to the cluster analysis (Fig. 2), both ncRNAs types responded differently to the salinity increase: asRNAs were mainly upregulated (cluster 1 and 2) while sRNAs were mainly downregulated (cluster 3 and 4). The salt-induced SRNA patterns, were again highly dynamic and mainly transient. For example, the SRNA IsaR1 was found to be transiently up-regulated in response to salt but returned to control levels in long-term salt acclimated cells (Suppl. Table S1). IsaR1 is a key player in the acclimation of the photosynthetic apparatus to iron starvation by targeting several mRNAs encoding $\mathrm{Fe}^{2+}$-containing proteins and enzymes involved in pigment and FeS cluster biosynthesis (Georg et al., 2017). In addition to its role in iron acclimation, IsaR1 controls directly the synthesis of a key enzyme in GG synthesis, GgpS at the post-transcriptional level early during salt acclimation (Rübsam et al., 2018).

Altogether, eleven SRNA candidates were found to be differentially expressed at all-time points, three candidates were up- and 8 were down-regulated (Suppl. Table S5). Their potential roles in the salt acclimation process is an interesting topic for future research.

\section{Global evaluation of the proteome in salt-acclimated cells}

Proteome analyses using data-independent acquisition mass spectrometry (HDMS ${ }^{\mathrm{E}}$ ) were performed with extracts from cells cultivated at either $0 \% \mathrm{NaCl}$ (control cultures) or $4 \% \mathrm{NaCl}$ (7 days' salt-acclimated cultures, Fig. 1). To improve coverage and to obtain additional information on cellular localization, the proteome of different fractions was analyzed: 1 . Total protein extracts obtained without any centrifugation; 2 . The debris fraction obtained after low speed centrifugation (yellowish brown pellet); 3. The soluble fraction representing the blueish supernatant after high-speed centrifugation; and, 4. The membrane-enriched fraction representing the washed green pellets after high-speed centrifugation (further details are described in the supplementary material). 
In total, 1816 proteins, approximately 52\% of the entire proteome of Synechocystis 6803 in the UniProt database, were identified by at least two tryptic peptides per protein (Suppl. Table S6). In particular, 1253 proteins were found in the total extracts and 823 in the soluble fraction, while 1608 and 1421 proteins were identified in the membrane-enriched and debris fractions, respectively (Fig. 4). Similar to previous reports on the Synechocystis 6803 proteome (Gao et al., 2015a), many proteins (79\%) were found in both, the soluble as well as the membrane fractions, although the membrane pellet was washed with high salt and high $\mathrm{pH}$ buffers. The occurrence of proteins in different fractions clearly correlates with their numbers of transmembrane helices (Suppl. Fig. S2). In the present study, we also investigated the cell debris that is usually removed from proteome analyses if cellular fractionation is performed. The vast majority (96.3\%) of proteins in this fraction was also identified in the membrane-enriched fraction. However, 29 proteins were exclusively found in the debris fraction, for example the large SIr1567 protein, a putative outer membrane protein (Suppl. Table S7). Many other proteins enriched in the debris fraction are annotated as components of the cell envelope or of the outer membrane-bound periplasmic space.

Salt-dependent changes in protein abundances were evaluated in total protein extracts as well as three subcellular fractions (Fig. 4). For the selection of differentially expressed proteins cut-off criteria were defined by a fold change of $\geq|1.5|$ and a corresponding p-value $<0.05$. However, for a large number of proteins less pronounced fold change values were also statistically significant. The volcano plots indicate that the group of stably up-regulated proteins (Suppl. Fig. S3) displayed larger fold changes than down-regulated proteins, resembling the observations made with the transcriptomic data (Suppl. Fig. S1).

Next, we analyzed whether or not particular proteins showed consistent salt-related changes in the different proteome fractions. For most proteins, well-matching values were found in the different fractions and in the total extract. However, in some cases the relative abundances showed an inverse relation in membrane and soluble fractions. For example, many ribosomal proteins occurred in lower amounts in the soluble fraction but were elevated in the membrane and debris fractions of salt-acclimated cells compared to control cells (Suppl. Fig. S4). Similar patterns were found, among others, for the subunits of the ATP synthase, the phycobilisome linker polypeptides, and the RNA polymerase. These results indicate cultivation-dependent differences in the distribution of proteins to the different cellular fractions as reported in other studies (Gao et al., 2015b; Pattanayak et al., 2020). In our case, some protein complexes such as ribosomes might be more stable under high salt conditions, resulting in their enrichment in the membrane fraction and depletion in the soluble fraction. To deal with this situation, we calculated a weighted fold change from the subcellular fractions data of each protein and showed that it was highly concordant with the corresponding fold change from the total protein extract (Suppl. Fig. S5; more detailed 
description is given in the supplementary material). Finally, the mean value of the weighted fold change from the subcellular fractions and the fold change from the total protein extract was used. If only one of these two values was available, it was used directly as final fold change. This led to a list of 1803 proteins, to which two membrane proteins were appended that are known (Slr0531) or suspected (SIl1037) to be important for salt acclimation, but were identified by individual peptides only. As a final result, 190 proteins were up-regulated and 189 protein down-regulated 7 days after salt shock among the 1805 quantified proteins 286 (Suppl. Table S6).

\section{Correlation analysis of salt-stimulated transcriptome and proteome}

A correlation analysis was performed to analyze the overall relation between transcriptomic ( $\log _{2}$ fold changes of mRNA after $24 \mathrm{~h}$ ) and proteomic changes ( $\log _{2}$ fold changes of protein abundances after 7 d). 1749 transcript/protein pairs could be matched (Fig. 5; Suppl. Table S8). The Pearson correlation coefficient for the proteomic and transcriptomic data sets was $r$ $=0.58$ indicating a quite good relationship, especially taking into consideration that sampling was done in different laboratories and at different time points. To grade the correlation between the newly acquired transcriptomic and proteomic data sets, we also compared our proteome data to the previously published transcriptome data sets by Marin et al. (2004) and Billis et al. (2014). In both cases, lower correlation coefficients of $r=0.41$ and $r=0.42$, respectively, were obtained. Next, we calculated the correlation coefficients between our transcriptome data and the transcriptome data of Marin et al. (2004) and Billis et al. (2014) using the same subset of mRNAs as for the comparison with the proteome. Surprisingly, the obtained coefficients of $r=0.49$ and $r=0.50$ showed a slightly lower correlation between different transcriptomic data sets than correlation between the proteomic and the present transcriptomic data set (Fig. 5C). These findings indicate that culture conditions significantly influence the comparability of different data. Nevertheless, in all cases a close correlation was observed for the expression of genes that are of direct importance for salt acclimation, while the expression of other genes can vary depending on small differences between the culture conditions leading to relatively low Pearson correlation coefficients.

\section{Detailed examination of specific processes}

\section{Compatible solute metabolism and transport constitutes a salt-specific stimulon}

309 Central to salt acclimation of Synechocystis 6803 is the accumulation of the main compatible solute GG, because mutants affected in the genes ggpS and ggpP (stpA) encoding the GG synthesis enzymes showed the highest degree of salt sensitivity compared to wild type

312 (Hagemann et al., 1997; Marin et al., 1998). As initially found by Reed and Stewart (1985), salt-acclimated cells accumulate high amounts of the compatible solute GG, which represents the by far largest pool of low molecular mass organic compounds. The amount of 
GG is approximately 2000times higher in salt-grown cells compared to the trace amounts of GG in control cells (Fig. 6C). The second compatible solute sucrose is approximately 1000fold less abundant $\left(0.28 \mathrm{nmol} O D_{750}{ }^{-1} \mathrm{ml}^{-1}\right)$ than $\mathrm{GG}$ in salt-acclimated cells (Suppl. Table S10), because it mainly plays a role as transiently accumulated osmolyte after salt shocks in Synechocystis 6803 (Kirsch et al., 2019).

Corresponding to the high GG accumulation, the GgpS and GgpP proteins and mRNAs showed significantly elevated levels (Table 2), which is supported for GgpS by Northern- and Western-blotting (Fig. 6AB). The sucrose synthesis enzymes Sps and Spp also exceeded the threshold for significant protein changes (Table 2), whereas the sps mRNA was only transiently increased (Suppl. Table S1) consistent with the transient accumulation profile of sucrose (Kirsch et al., 2019). The ggpS downstream gene glpD encoding glycerol 3phosphate dehydrogenase, which is involved in the synthesis of the GG precursor glycerol 3phosphate (G3P) from dihydroxyacetone phosphate, is also salt-stimulated. Overlapping with the ggpS promoter region exist the small ORF ss/3076 that encodes for the ggpS repressor GgpR (Klähn et al., 2010b), which could not be detected in the proteome. Upstream of ggpS on the opposite strand, the genes for the GG hydrolase (GghA) and glycerol kinase (GlpK), the latter is involved in synthesis of the GG precursor G3P from glycerol, are located. They show similar expression pattern as ggpS. In contrast to the salt induction of genes for G3P synthesis, the glgC gene encoding the enzyme for ADP-glucose synthesis, the second precursor for GG, is not salt-regulated on RNA or protein levels.

Immediately downstream of $g g p P$, the salt-induced GgtA protein is encoded that acts as the ATP-binding subunit of the GG transporter (Ggt). The genes for the other Ggt subunits, GgtB, C and D (two of them were identified among the salt-stimulated proteins) form a separate salt-induced operon. The co-regulation of genes and proteins for GG synthesis and the ABC-type osmolyte transporter Ggt, which all belong to the cluster 2 (Table 2), indicates their functional cooperation in salt-acclimated cells, in which the transporter is mainly responsible for the avoidance of GG (and sucrose) leakage from the cells (Mikkat and Hagemann, 2000). However, the GG hydrolase GghA (SIr1670), which degrades GG into glucose and glycerol after hypo-osmotic treatments (Kirsch et al., 2017), showed also enhanced expression on mRNA and protein level in salt-acclimated cells. It has been discussed that the GG synthesis and degradation are mainly regulated at enzyme activity level that are differentially affected by cellular ion contents; elevated internal ion content leads to biochemical activation of GG synthesis and inactivation of GG cleavage (Kirsch et al., 2019). Hence, the increased amounts of putatively biochemically inactive GghA protein likely prepares Synechocystis 6803 cells for sudden decreases in osmolarity. It should be noted that the GgpP and GlpD proteins involved in GG synthesis were not detected in the soluble but exclusively in the membrane-associated fraction. This localization could indicate 
that these proteins might be involved in additional processes such as fatty acid (GlpD) or EPS (GgpP) biosyntheses (Kirsch et al., 2017).

Finally, the salt-induced proteins involved in GG metabolism and transport as well as in G3P synthesis are obviously part of a larger group of salt-induced genes/proteins in Synechocystis 6803 , which presumably can form a salt-regulon. Using ggpS as search string in the CyanoExpress database, which compiles gene expression data sets from Synechocystis 6803 (Hernandez-Prieto and Futschik, 2012), revealed more than 30 genes showing similar expression pattern under different environmental stimuli (Suppl. Fig. S6). The occurrence of all genes related to GG biochemistry, which are proven to be involved in salt acclimation, make it very probable that several of the co-regulated genes encode proteins also specific for this stress acclimation. This assumption is supported by our finding that many of these proteins also accumulated to higher levels in salt-acclimated cells. In cases where the protein levels were not significantly elevated, the corresponding gene showed only transient stimulation at the earliest time points after salt addition (Suppl. Fig. S6).

\section{Transporters and channels belonging to the salt-specific stimulon}

Clustering and functional enrichment analyses clearly indicated that differential regulation of proteins related to membrane transport is generally an important mechanism for salt acclimation (Suppl. Table S9). Among them, the mechanosensitive channel protein of small conductance (MscS) Slr0639 accumulated to the highest level, fold change of 14.3 (RNA approximately 2-fold) (Suppl. Table S6; Fig. 7). Two other MscS proteins, Slr0765 and SIl1040 were identified at elevated protein and RNA levels as well. Msc proteins are important for proper acclimation to hypo-osmotic treatments to facilitate quick release of compatible solutes and to prevent burst of cells (Levina et al., 1999). Hence, similar to elevated GghA, the MscS accumulation likely prepares the cell for upcoming events of lower osmotic pressure as safety valves as has been also shown in many heterotrophic bacteria (Perozo et al., 2001; Stokes et al., 2003). In contrast, the abundance of MscL (Slr0875), which is involved in water movements after sudden osmotic shocks on Synechocystis 6803 (Shapiguzew et al., 2005; Azad et al., 2011), did not change in long-term salt-acclimated cells.

$\mathrm{Na}^{+} / \mathrm{H}^{+}$antiporters are considered as main exporters of excess $\mathrm{Na}^{+}$and, therefore, play a crucial role for salt acclimation (Hagemann, 2011). The Synechocystis 6803 genome codes for six $\mathrm{Na}^{+} / \mathrm{H}^{+}$antiporters, five of them were identified in the proteome. Among them, the protein abundance of NhaS2 and NhaS5 was significantly enhanced in salt-acclimated cells (Fig. 7), whereas the amount of NhaS3, which is essential for cell viability and was discussed to be mainly responsible for $\mathrm{Na}^{+}$export (Wang et al., 2002; Elanskaya et al., 2002), remained 
unchanged (Suppl. Table S6). However, the fact that the abundance of a protein remains unchanged does not exclude an essential function of this protein for salt acclimation, because it could be regulated on biochemical level according to cellular demands. While crucial roles were assigned to NhaS2 under low $\mathrm{Na}^{+} / \mathrm{K}^{+}$ratios (Mikkat et al., 2000) or growth at different $\mathrm{pH}$ values (Wang et al., 2002), the nhaS5 mutant did not show any changes compared to wild type (Wang et al., 2002; Elanskaya et al., 2002). Unfortunately, none of the previously discussed candidates for chloride exporters (SIl1864, Slr0753, SII0855; Hagemann, 2011) could be identified in our proteome data set. Among them, only the gene sll1864 was transiently stronger expressed on mRNA level in salt-shocked cells (Suppl. Table S1).

In addition, several other proteins potentially involved in ion transport were found in higher abundances in salt-acclimated cells (Fig. 7). For example, two of the 7 annotated cationtransporting ATPases (Wang et al., 2002) were elevated in the proteome (SIr1950 with FC 1.8 and SII1614 with FC 1.68) and two hours after salt shock in the transcriptome as well. Furthermore, the amount of the protein SIr1257, which comprises a ligand-gated ion channel domain, increased 4-fold, whereas the corresponding mRNA was slightly below the threshold of 2-fold in salt-acclimated cells. Many other transport proteins such as Slr0798 (Zinctransporting ATPase, FC 1.64), Sll0615 (GDT1-like, possible $\mathrm{Ca}^{2+} / \mathrm{H}^{+}$antiporter, FC 1.59) or those involved in uptake of nutrients such as nitrate, phosphate, or magnesium were also present in higher amounts in salt-acclimated cells (Suppl. Tables S6, S9). Finally, several transporters involved in iron uptake are transiently up-regulated at mRNA level, which indicates that the transient influx of high $\mathrm{NaCl}$ amounts in the cells somehow interferes with the general ion homeostasis including iron availability. However, there expression returned to control mRNA as well as protein levels after long-term salt acclimation (Fig. 7).

\section{Many salt-induced proteins are involved in general stress response}

Two proteins of unknown function, Sll1862 and sll1863 showed 9.9- and 13.3-fold, respectively, higher abundances in salt-acclimated cells (Table 3). Searches using CyanoExpress (Hernandez-Prieto and Futschik, 2012) revealed that these proteins are induced on mRNA level not only after salt stress but also in response to many other stress treatments, hence, they belong to the group of general stress proteins. The Sll1863 protein was previously identified as the top salt-induced gene/protein (Fulda et al., 2006), while in the present study it shares the top four positions with two MscS proteins, which were not previously quantified. The induction of SIl1862 and SIl1863 also led to high total protein amounts in salt-acclimated cells, both of them belong to the 60 most abundant proteins. However, their inactivation by interposon mutagenesis did not result in a salt-sensitive phenotype (unpublished results of Hagemann group). 
Several heat-shock proteins that are involved in protein folding and repair have been previously identified among the salt-induced, general stress proteins (Fulda et al., 2006). In the present study, only the $33 \mathrm{kDa}$ chaperone (SIl1988) was more than 2-fold accumulated while its mRNA showed only a slight increase, whereas the amounts of DnaK, GroEL, or DnaJ proteins were not significantly changed. Moreover, the small, $16.6 \mathrm{kDa}$ heat shock protein (SII1514) was decreased despite its RNA accumulated after the salt addition (Table 3), whereas it was found before in significant enhanced amounts in salt-acclimated cells (Fulda et al., 2006). These differences most probably result from different cultivation conditions and sampling times in the different studies. Many other salt-stimulated proteins have no functional annotations, however, the proteins SIr0967, SIr2019, SII0528, and SII0947 were all also implicated in multiple stress responses of Synechocystis 6803 (Uchiyama et al., 2014; Matsuhashi et al., 2015; Lei et al., 2014; Galmozzi et al., 2016).

Moreover, an overlap between salt stress and iron-starvation response has been often observed, because in previous studies many genes/proteins serving as markers for ironstarvation have been observed in elevated amounts in salt-stressed cells as well (Marin et al., 2004; Fulda et al., 2006). In the present study, many proteins related to iron transport are found at higher mRNA levels in the early time points (Fig. 7, Suppl. Table S1). However, in long-term acclimated cells only two iron-regulated proteins are significantly elevated (Table 3). The general stress protein SIr1894, which is annotated as MrgA or Dps like protein, was found in higher abundances in salt-acclimated cells. It has been shown to be involved in oxidative stress response (Li et al., 2004) or in the mobilization of iron-storage after transfer from iron-replete into iron-deplete conditions (Shcolnick et al., 2007). Furthermore, flavodoxin (isiB) is clearly accumulated, which plays an important role in the salt-stimulated photosynthetic cyclic electron transport (Hagemann et al., 1999).

\section{Salt effects on proteins involved in the basic cell physiology}

449 Previous transcriptome analysis revealed that genes encoding subunits of protein complexes are co-regulated in salt-stressed cells (Marin et al., 2004). The evaluation of the proteome data showed that the abundances of most proteins belonging to the photosystem (PS) I and

452 II, phycobilisomes, ribosomes, or enzymes of the tricarboxylic acid (TCA) cycle were slightly 453 lower after long-term salt acclimation (Fig. 8). Only a few exceptions were found. The Psb28 454 protein of PSII was elevated (FC 1.8). This subunit is involved in PSII assembly and repair 455 (Nowaczyk et al., 2012), thus its higher amount could indicate that the PSII is less stable in 456 salt-acclimated cells. As another example, the transcript levels of sll1471 encoding the 457 CpcG2 phycobilisome rod-core linker polypeptide was strongly reduced in the microarray dataset upon short term salt stress and exhibited a lowered protein level ( $\log _{2}$ FC of -0.42) as well. CpcG2 is the rod core linker of the smaller CpcL-phycobilisome type (Kondo et al., 
460

461

462

463

464

465

466

467

468

469

470

471

472

473

474

475

476

477

478

479

480

481

482

483

484

485

486

487

488

489

490

491

492

493

494

495

2005; Liu et al., 2019b), which has been implicated in the formation of a PSI/NDH supercomplex in Synechocystis 6803 (Gao et al., 2016). Furthermore, alpha phycocyanobilin lyase (CpcF, sll1051), a protein involved in phycobiliprotein assembly into phycobilisomes, increased 1.7-fold. Another phycocyanobilin lyase (CpcE, s/r1878) was also increased. Finally, many ribosomal proteins showed a tendency to slightly lowered amounts (Fig. 8), which correlates with the slower growth of salt-acclimated cells (Hagemann et al., 1994). The results show that cells acclimated to $684 \mathrm{mM} \mathrm{NaCl}$ for 7 days have reached a new steady state, in which many basic physiological processes differ from the control cultures. Regarding basic cellular processes our current transcriptome data of salt-shocked cells after $24 \mathrm{~h}$ showed a high agreement with the proteome data.

The potential impact of high salinity on the cell surface of Synechocystis 6803 cells is indicated by the observation that 8 salt-induced proteins were found among the 19 identified proteins of the functional category "Murein sacculus and peptidoglycan“. These proteins include MurF (SIr1351), DacB (SIr0804), MurA (SIr0017), MurG (sIr1656) and MItA (SII0016), which all are enzymes probably involved in cell wall or cell envelope biogenesis (Suppl. Table S6). Their coordinated up-regulation indicates that salt stress induces a reorganization of cell wall structures, which possibly decrease its permeability for inorganic ions. Changed abundances of several further proteins involved in the reorganization of the cell surface support this assumption. The lowered abundance of WZC (SII0923) is consistent with the lowered amount of EPS of salt-acclimated cells (Kirsch et al., 2017). Some of the most giant proteins encoded in the Synechocystis 6803 genome that are believed to have functions in the cell envelope, such as the $192 \mathrm{kDa}$ protein SII0723 (FC 3.9) and the $214 \mathrm{kDa}$ protein Sll1265 (FC 1.6) accumulated in salt-acclimated cells. However, in contrast to the proteins participating in salt-regulated compatible solute synthesis or ion transport, a lower correlation between mRNA and protein abundances was found for the proteins involved cell envelope related processes.

\section{Differential expression of regulator proteins}

Regulatory proteins are highly important for stress acclimation, but were underrepresented in the proteome data. Only three out of 86 identified regulatory proteins showed significantly elevated protein but not increased mRNA levels in our study. All three represent different members of two-component regulatory systems (SIl1624 - Rre18, SIr1324 - Hik 23, SIr6110 - Rre on plasmid pSYSX). However, none of them is functionally characterized and none of the previously identified salt-stress-associated two-component regulators (Marin et al., 2003; Shoumskaya et al., 2005) showed higher protein amounts here. Further 15 proteins among the 86 identified regulatory proteins were found with lower abundances in salt-acclimated cells, mostly in concordance to their mRNAs. 
The list of genes co-regulated with ggpS and other genes/proteins for the GG biochemistry included one transcription factor, PrqR (Slr0895; Suppl. Fig. S6). Hence, it is tempting to speculate that PrqR might be involved in the salt-stimulated expression of $g g p S$ and other genes in this putative regulon. PrqR was reported as repressor for genes involved in glucose metabolism and oxidative stress acclimation (Khan et al., 2016). The LexA protein, which is not changed on protein level but became significantly decreased on RNA level (Suppl. Fig. S7), has been shown to act as negative regulator of GgpS and GgpP expression (Takashima et al., 2020). However, the fact that the abundance of a protein remains unchanged does not exclude an important function of this protein for salt acclimation, because especially

505

506

507

508

509

510

511

512

513

514

515

516

517

518

519

520

521

522

523

524

525

526

527

528

529

530

531 regulatory proteins are often activated/inactivated upon association with other proteins or metabolic signals. Moreover, the expression of genes for other annotated transcription factor changed. For example, the gene sll0998 encoding the transcription factor RbcR, which regulates the expression of the key $\mathrm{CO}_{2}$-fixing enzyme ribulose 1,5 bisphosphate carboxylase/oxygenase (RubisCO), showed lower abundances on protein and RNA levels in salt-acclimated cells. Albeit not passing our significance criteria, both the large and small subunit of RubisCO were reduced by $20-30 \%$ that could indeed result in lower $\mathrm{CO}_{2}$-fixing activity given the high amount of RubisCO protein per cell.

Finally, sigma factor cascades have been implicated in the stress acclimation of bacteria. Among the down-regulated genes as well as proteins is the anti-sigma $\mathrm{F}$ factor antagonist Slr1859 (Suppl. Fig. S7), whose homolog in Bacillus subtilis is involved in posttranslational regulation of Sigma factor F (Clarkson et al., 2004). Interestingly, the Sigma F (SIr1564), which itself showed an unchanged protein abundance, is one of the candidate factors involved in mediating the salt-dependent expression of ggps (Marin et al., 2002). Other sigma factors, particularly the genes encoding SigB and SigC were transiently induced after salt addition, while the amount of SigC slightly decreased in the proteome (SigB not identified) (Suppl. Fig. S7). Mutations of these group 2 sigma factors have been shown to reduce the stress tolerance including salt resistance of Synechocystis 6803 (Tyystjärvi et al., 2013). Hence, the salt-dependent activity of different sigma factors might significantly contribute to the observed salt-induced expression changes on transcript and protein levels.

\section{Salt stress differentially affects chromosome regions}

Most salt-regulated proteins are encoded by single genes or in small operons that are spread on the Synechocystis 6803 chromosome. However, some salt-regulated genes are clustered on specific chromosomal regions. The most remarkable examples are clusters including the genes ggpS and ggpP, which were found in regions on the chromosome comprising several genes/proteins with salt-stimulated expression. The ggpS cluster comprises 9 salt-regulated genes, which are at least transiently stronger expressed in salt-grown cells than in control 
532 cells (Suppl. Fig. S8). Upstream of the ggpS/g/pD operon, on the opposite strand, a salt533 regulated gene cluster of at least 7 genes (s/r1670-1677) is found. This gene organization is 534 widely conserved in the genomes of many GG-accumulating cyanobacteria (Kirsch et al., 535 2017). A second salt-stimulated gene cluster can be found downstream of ggpP and ggtA, 536 which comprises 5 different genes (Suppl. Fig. S9). In addition to glpK four other genes 537 (three encode subunits of protochlorophyllide synthase, e.g. ChIN, slr0750; see Table 3) are 538 at least transiently salt-regulated but have not yet shown to code for proteins directly involved 539 in salt acclimation.

540 Another salt-stimulated gene cluster was found on the plasmid pSYSA. The genes s/l7063541 sll7067 (for one example see Table 3) are cas genes that encode structural proteins of one 542 of the three CRISPR-Cas systems in Synechocystis 6803, called CRISPR2 (Scholz et al., 543 2013). The five proteins were significantly up-regulated whereas the transcripts were 544 transiently down-regulated and then slightly up-regulated after $24 \mathrm{~h}$ of salt acclimation. In 545 contrast, the CRISPR3 system (s/l7085-s/l7090) on the same plasmid was consistently 546 down-regulated at RNA and protein level. Recently, it has been shown that these proteins 547 form a stable protein complex together with their cognate crRNAs (Riediger et al., 2021).

548 There are also some examples of coordinately down-regulated genes/proteins in salt549 acclimated cells. One example is the large region (chromosome positions 1181250 550 1200000) comprising 22 genes, which are forming three operons (Suppl. Fig. S10). The first 551 operon s/r1406-1410 encodes proteins of unknown function. The second salt-repressed 552 operon is situated on the opposite strand (s/l1307-1304 and s/l1784-1785) and also mostly 553 encodes not functionally annotated proteins; however, the protein SIl1305 resembles ketose 554 3-epimerases while SII1307 and SII1784 are predicted outer membrane-bound periplasmic 555 proteins. Hence it can be speculated that these proteins are somehow involved in cell wall 556 synthesis/reorganization. The third salt-repressed operon in this region comprises the genes 557 slr1852-1862. This cluster contains several annotated genes (SIr1855 N-acetylglucosamine 558 2-epimerase, SIr1856 - anti-sigma factor antagonist, GlgX1 - glycogen branching enzyme 1, 559 IcfG - carbon metabolism regulator), which play important roles in the primary carbon 560 metabolism and, particularly, its regulation (Beuf et al., 1994; Shi et al., 1999). Another 561 example of coordinated down-regulated genes/proteins represents the large operon s/r0144562 0152, which has been noted before as one of the highly coordinated expressed regions on 563 the chromosome of Synechocystis 6803 (e.g., Summerfield and Sherman, 2008) that is 564 controlled by the redox-responsive transcription factor RpaB (Riediger et al., 2019).

\section{Metabolome analysis of salt-acclimated cells}

566 The presence of high $\mathrm{NaCl}$ amounts in the medium induces a massive $\mathrm{GG}$ accumulation 567 (Fig. 6C), which likely triggers a strong redistribution of organic carbon in Synechocystis 
6803. The large impact of GG synthesis on overall carbon metabolism is also consistent with the observation that many proteins (and their genes) involved in glycogen metabolism as well as glycolysis showed significant changes in their abundances (Table 4). For example, the neopullulanase, glycogen phosphorylase GlgP2 (SIr1367) and one debranching enzyme GlgX1 (SIr0237, the other one Sll1857 is decreased) showed higher abundances on protein as well as RNA levels in salt-acclimated cells. The different response of the two GlgX proteins towards salt stress has been previously shown with Western-blotting (lijima et al., 2015). Hence, the demand of organic carbon for the synthesis of GG precursors is at least partly supported by an enhanced glycogen breakdown and reduced glycogen build up, because glycogen and GG synthesis are competing for the same precursor, ADP glucose. The relatively low carbon/nitrogen state in salt-acclimated cells is also reflected by the lowered amount of 2-oxoglutarate (2OG, Fig. 9), which is the key metabolic signal reporting changes of the cellular carbon/nitrogen ratio in cyanobacteria (Hagemann et al., 2021).

To obtain a snapshot on metabolites of the central carbon and nitrogen metabolism, LCMS/MS was used (Suppl. Table S10). The relative levels of the RubisCO carboxylation and oxygenation products 3PGA and 2PG, respectively, showed opposite behavior (Fig. 9). 3PGA accumulated approximately 3-fold less in salt-acclimated cells, while 2PG was clearly enhanced. This could indicate a decreased $\mathrm{CO}_{2} / \mathrm{O}_{2}$ ratio at the active site of Rubisco, since the amounts of these gasses mainly regulate its relative carboxylation/oxygenation activity. For example, it might be possible that due to the higher content of inorganic ions inside the salt-exposed cells carboxysomes are less gas tight in high salt-grown cells, thereby promoting a better diffusion of oxygen into carboxysomes reducing the $\mathrm{CO}_{2} / \mathrm{O}_{2}$ ratio. The observed changes in the 3PGA and 2PG levels are consistent with the reported lower photosynthetic activity and growth rate in salt-acclimated cells of Synechocystis 6803 (e.g., 592 Hagemann et al., 1994), which certainly also reduce Calvin-Benson-cycle activity. 593 Consistently, the protein abundances of photosynthetic complexes, Calvin-Benson-cycle enzymes including RubisCO and components of the cyanobacterial inorganic carbonconcentrating mechanism were found at $10-40 \%$ lower levels, which is below our significance threshold but might contribute to lower photosynthetic activity in salt-acclimated cells.

597 Furthermore, more organic carbon could be taken out from the Calvin-Benson-cycle, which is 598 for example seen in the increased amount of pyruvate and organic acids in the reductive branch of the TCA cycle, such as malate and fumarate. This interpretation is also supported 600 by the finding that Gap1, the glyceraldehyde dehydrogenase 1 (Slr0884) involved in sugar 601 catabolism (Koksharova et al., 1998) is also up-regulated (Table 4), while Gap2 involved in 602 photosynthetic carbon assimilation did not change (Suppl. Table S1).

603 In addition to the carbon fixation and allocation, nitrogen assimilation is altered in salt604 acclimated cells, which is reflected by enhanced glutamine and glutamate levels while 20G, 
the carbon skeleton used for ammonia assimilation decreased (Fig. 9). Increased glutamate levels have been often reported in salt-exposed bacteria (Hagemann, 2011), because this negatively charged amino acid is compensating the positive charge of cations, especially $\mathrm{K}^{+}$. The enzymes involved in the GS/GOGAT cycle for assimilation of $\mathrm{NH}_{4}{ }^{+}$into $2 \mathrm{OG}$ did not significantly change their expression in long-term salt acclimated cells, but were significantly lowered immediately after salt addition. However, the glutamate decarboxylase Gad (SIl641) showed increased expression in salt-grown cells. Proline, which is often used in heterotrophic bacteria as compatible solute and can be synthesized from glutamate, is not changed during salt acclimation of Synechocystis 6803 (Fig. 9). Moreover, aspartate and arginine levels decreased. These amino acids serve as precursors for cyanophycin, the nitrogen storage compound of Synechocystis 6803. In this regard it is interesting to note that cyanophycin synthetase (SIr2002, FC 0.64) was decreased on protein level while the mRNA did not change. Furthermore, the mutation of a gene presumably involved in cyanophycin turnover resulted in a salt-sensitive phenotype of Synechocystis 6803 (Zuther et al., 1998). These observations also add to the assumption of altered nitrogen assimilation in salt-loaded cells. Marked changes in the amino acid composition had been also reported for Synechocystis 6803 cells when grown in artificial seawater medium compared to BG11 (lijima et al., 2015). Similarly, global changes in the carbon- and nitrogen metabolism have been noticed in Synechococcus sp. PCC 7002 (Aikawa et al., 2019).

\section{Discussion}

\section{Overall correlation of omics data}

Our proteomic and transcriptomic data showed a good relationship (Pearson correlation coefficient, 0.58) especially taking into consideration that sampling was done in different laboratories and at different time periods after salt treatment. This finding indicates that in most cases transcriptional activation/repression leads to enhanced/diminished protein amounts, whereas the differential regulated ncRNAs rather regulate single genes/proteins. Generally, relationships between the transcript and corresponding protein amounts are influenced by several processes, such as (1) impact of mRNA processing or ncRNAs on translation rates, (2) protein's half-life, and (3) protein synthesis delay (Liu et al., 2016). Other studies found widely differing correlation coefficients between cyanobacterial transcriptomes and proteomes depending on the conditions examined. For example, experiments with Synechocystis 6803 cells shifted from high to low $\mathrm{CO}_{2}$ conditions showed also a good correspondence between transcriptomics and proteomics (Spät et al., 2021), whereas Toyoshima et al. (2020) reported a rather low correlation between transcriptomics and proteomics in Synechocystis 6803 cells grown under phototrophic, mixotrophic or 
640 heterotrophic conditions. Similarly, a very low correlation was reported from an integrated 641 proteomic and transcriptomic analysis of salt stress responses in Synechocystis 6803 (Qiao 642 et al., 2013).

643 Moreover, the comparison of proteome and transcriptome data from different studies, despite 644 the varying degree of correspondence, offers the possibility to filter out regularly, truly salt645 regulated proteins. To this end, we compared three transcriptomic with our proteomic data 646 sets to identify further proteins potentially involved with unknown function in salt acclimation. 647 Filtering the four datasets for features that were at least 1.5 -fold increased $\left(\log _{2}>0.58\right)$ in 648 the three transcriptome data sets and at least 1.3-fold increased $\left(\log _{2}>0.38\right)$ in the 649 proteome data set produced a list of 44 genes, for which in 25 cases the corresponding 650 proteins were identified (Suppl. Table S11). This group of genes/proteins includes 11 that are 651 involved in GG metabolism and ion transport. Among the rather general stress proteins with 652 unknown function are the SII1862 and SIl1863 proteins as well as the putative zinc 653 metalloprotease SII0528 and the SIr1894 protein, which all have been discussed before. 654 Other proteins with unknown function in salt acclimation such as the methionine 655 aminopeptidase B (MAP B, Slr0786; co-expressed with ggpS, Suppl. Fig. S6), SII0723 and 656 Slr0001 are candidate proteins for further studies. Among the 19 salt-induced genes, whose 657 proteins were not identified in our study, only the s/r0530 gene product as component of the 658 GG transporter is functionally related to known processes in salt acclimation.

659 The good correlation of transcriptomic and proteomic changes extends to the alterations on 660 the metabolome level. Basic alterations in the central carbon and nitrogen metabolism are also supported by expression changes. However, biochemical alteration due to changed metabolite and ion levels on key enzyme activities certainly contribute to the novel metabolic 663 homeostasis in salt-acclimated cells as has been recently discussed for the metabolic acclimation of Synechocystis 6803 towards different $\mathrm{CO}_{2}$ availability (Jablonsky et al., 2016).

Salt shock leads to a temporally staggered reshaping of the transcriptome

\section{composition}

667 The salt acclimation response goes way beyond the induction of gene expression required 668 for the compatible solute machinery, it has clearly a great impact on general metabolism. 669 Along the temporal axis, the reprogramming of gene expression can be differentiated 670 between an early response with the respective minima and maxima leading to the four 671 different clusters (Fig. 2). The metabolic response included the rapid repression of the 672 ammonia assimilation system, detected by decreased transcript abundances for amt1 and 673 gInA encoding the ammonium transporter and the primary enzyme for ammonium 674 incorporation, glutamine synthetase (GS). Consistently, increased transcript levels were 675 found for genes gifA and gifB, which encode inhibitory proteins for GS, thereby blocking 
676 ammonium assimilation (for an overview see Bolay et al., 2018). After $24 \mathrm{~h}$ the transcript

677 levels of these genes were at the initial levels.

678 The question arises, which processes are responsible for the staggered reshaping of the 679 transcriptome. It has been shown that shortly after salt addition the cytoplasmic composition 680 underwent rapid changes due to ion and water movements, whereby the early high internal

681

682

683

684

685

686

687

688

689

690

691

692

693

694

695

696

697

698

699

700

701

702

703

704

705

706

707

708

709

710

711 ion contents, especially of $\mathrm{Na}^{+}$are discussed to inhibit metabolic activities but also to trigger acclimation responses such as GG synthesis activation (reviewed in Hagemann, 2011). The transporters responsible for the rapid ion movements are largely unknown, especially verified candidates for $\mathrm{Cl}^{-}$export are still missing. In the present study we did not find marked expression changes for genes encoding potential anion exporters. It can be assumed that these transporters are mainly regulated on their activity levels to manage the ion regulation within the first minutes to hours after salt shock, because de novo protein synthesis is one process clearly down-regulated after salt shock (Hagemann et al., 1994). However, we found that especially in the early time points after salt shock multiple ncRNAs become up- or downregulated, which were not covered in the previous transcriptomic datasets. These small RNAs likely reshape mainly the translational efficiency of specific target mRNAs. Hence, it is well possible that many of the identified asRNAs and SRNAs are important to fine tune the translational response in the acute stress situation. Further work is necessary to identify the specific targets of the sRNAs and to verify the action of the ncRNAs during salt acclimation in cyanobacteria.

\section{Impact of salt on DNA structure}

The identification of chromosomal regions in the Synechocystis 6803 genome with coordinately up- or down-regulated genes under high salt conditions indicates that the DNA structure likely differs between distinct chromosomal regions (Suppl. Figs. S8-10). Similar observations were made when the impact of antibiotics that affect DNA supercoiling was analyzed on global gene expression patterns in E. coli and Synechocystis 6803. Interestingly, a large overlap has been observed between the antibiotic-induced gene expression changes and the salt and osmostress responses (Cheung et al., 2003; Prakash et al., 2009). Hence, the salt-stimulated expression of genes in some chromosomal regions could be related to a relaxed DNA topology permitting easier access of the transcription machinery and vice versa. In this regard it is interesting to note that the gene s/r2058 encoding the DNA topisomerase $\mathrm{I}$ is higher transcribed in the first hours after salt shock (Suppl. Tables S1 and S2). The ATP-dependent DNA topoisomerases relax negative supercoils and are specifically involved in chromosome partitioning, which has been shown to be of fundamental importance for bacterial gene expression (Dorman and Dorman, 2016). Recently, another subunit of DNA toposiomerase has been suggested to be involved in the 
regulation of DNA replication in a mutant defective in one of the dominating DNA methylation activities in Synechocystis 6803 (Gärtner et al., 2019). It might be possible that differences in DNA methylation and thereby induced changes in the DNA/protein association are at least partly responsible for the different accessibility of specific chromosomal regions under different salt conditions. Finally, the interaction of chromosomal DNA with the GG synthesizing enzyme GgpS has been shown to be central for the ion-mediated activation/inactivation of its biochemical activity (Novak et al., 2011). Collectively, our data support the notion that changes in DNA structure and DNA/protein interactions due to altered ionic and electrostatic relations play an important role in microbial salt acclimation.

\section{Future developments - regulation and application}

One still open question is how salt stress is sensed and transduced to the cellular gene expression machinery. Despite several efforts, specific salt-sensing proteins have not been identified in cyanobacteria. The screening of mutant collections defective in histidine kinases and cooperating response regulators identified some two-component systems that are involved in the salt-induced regulation of different general stress proteins, however, the induction of salt-specific proteins including ggpS remained unchanged (Marin et al., 2003; Shoumskaya et al., 2005). Two proteins were identified as repressors for ggpS, the small GgpR protein (Klähn et al., 2010b) and the transcriptional factor LexA have been shown to bind specifically the ggpS promoter (Takashima et al., 2020). However, the role of LexA as specific salt-sensing transcription factor is unlikely, because it is also involved in the regulation of many other processes in Synechocystis 6803, for example a verified role in fatty acid accumulation (Kizawa et al., 2017), and the lexA mutant has no reported salt-sensitive phenotype or changed GG accumulation. The present study identified two other likely candidates for a salt-stress specific gene regulation. First, the transcription factor PrqR (Slr0895) represents an interesting candidate, because the gene s/0895 is clearly coregulated with many genes coding proteins involved on GG metabolism (Suppl. Fig. S6). It has been recently shown that PrqR is involved in the acclimation to oxidative stress in Synechocystis 6803 (Khan et al., 2016). Salt stress is also inducing oxidative stress in cyanobacterial cells, hence, the finding of the role of PrqR in this stress acclimation process might of secondary importance. Second, the gene ss/1326 that possibly encodes a CopG

742 family transcription factor has been found strongly induced after salt shock in the DNA 743 microarray data set (Suppl. Table S1). Further work is necessary to validate whether PrqR or 744 CopG are somehow acting as salt-specific gene expression regulators. In the moment, it 745 might well be possible that ion-mediated changes in the DNA structure, RNA-polymerase 746 affinity, and enzyme activities might be the main and sufficient mechanism to acclimate towards different salt conditions in euryhaline bacteria such as Synechocystis 6803. 
Furthermore, salt acclimation is becoming more important for applied research with cyanobacteria regarding the direct use of compatible solutes as well as mass cultivation in sea water to make the process more sustainable (Pade and Hagemann, 2014; Cui et al., 2020). For example, the cyanobacterial production of mannitol (Wu et al., 2020) and trehalose (Qiao et al., 2020) has been promoted by cultivation at enhanced salinities. Moreover, a more salt-tolerant version of the fast-growing Synechococcus elongatus strain UTEX2973 has been engineered by the expression of GG synthesis genes, which can be used for biotechnological purposes in full marine waters (Cui et al., 2021) However, saline conditions might also negatively affect the production titer. The large impact of GG accumulation on the overall carbon metabolism also negatively influenced the ethanol production with Synechocystis 6803 at $4 \% \mathrm{NaCl}$ while $2 \% \mathrm{NaCl}$ were slightly stimulatory (Pade et al., 2017). Salt-regulated, strong promoters might be an option to improve transgene expression in salt-grown cyanobacteria. Hence, a deeper knowledge on salt acclimation will promote future biotechnological applications with cyanobacteria.

\section{Material and Methods}

\section{Cultivation and sampling}

764 Synechocystis sp. 6803 substrain PCC-M was used in all experiments. Axenic cells were maintained on agar plates with BG11 mineral medium at $30^{\circ} \mathrm{C}$ under constant illumination. For salt stress experiments, axenic cells were grown photoautotrophically in glass tubes containing liquid BG11 medium (TES-buffered at $\mathrm{pH}$ 8) at $29^{\circ} \mathrm{C}$ in the cooperating laboratories. If not stated differently, cultures were aerated with $\mathrm{CO}_{2}$-enriched air $\left(5 \% \mathrm{CO}_{2}\right.$ $[\mathrm{v} / \mathrm{v}]$ ) and kept under continuous illumination of $150 \mu \mathrm{mol}$ photons $\mathrm{m}^{-2} \mathrm{~s}^{-1}$ (warm light, Osram L58 W32/3). Control cells were cultured in NaCl-free BG11 medium, whereas salt-acclimated cultures were obtained after long-term growth (up to one week) in BG11 medium supplemented with $684 \mathrm{mM} \mathrm{NaCl}(4 \% \mathrm{NaCl}[\mathrm{w} / \mathrm{v}])$. For this, cells were transferred daily to fresh media with $4 \% \mathrm{NaCl}$. Short-term salt shock experiments were performed by adding crystalline $\mathrm{NaCl}$ to a final amount of $4 \%$ into control cultures at time point zero.

775 Subsequently, cells were harvested at defined time points.

776 For proteomics, cells were harvested by centrifugation at $14,000 \mathrm{~g}$ and $4^{\circ} \mathrm{C}$ for $5 \mathrm{~min}$. The 777 cell pellets were frozen in liquid nitrogen and stored at $-80^{\circ} \mathrm{C}$. For metabolomics, cells were 778 harvested by quick filtration on nitrocellulose filters $(0,45 \mu \mathrm{m}$ pore size) in growth light within $77930 \mathrm{~s}$. Cells on filters were then frozen in liquid nitrogen and stored at $-80^{\circ} \mathrm{C}$. For 780 transcriptomics, cells from $40 \mathrm{ml}$ culture were harvested by quick filtration on hydrophilic 781 polyethersulfone filters (Pall Supor-800, $0.8 \mu \mathrm{m}$ ), immediately immersed in $1 \mathrm{ml}$ of cold PGTX solution (Pinto et al., 2009) and frozen in liquid nitrogen. 


\section{Transcriptomic methods}

784

785

786

787

788

789

790

791

792

793

794

795

796

797

798

799

800

801

802

803

804

805

806

807

808

809

810

811

812

813

814

815

816

817

\section{RNA extraction}

Total RNA was extracted as described previously (Hein et al., 2013). Prior to the microarray analysis, $10 \mu \mathrm{g}$ of total RNA were treated with Turbo DNase (Invitrogen) according to the manufacturer's protocol and precipitated with ethanol/sodium acetate. RNA quality was verified by electrophoresis on MEN-buffered 1.5\% agarose gels supplemented with $6 \%$ formaldehyde.

\section{Microarray analysis}

To enable the comparison with a previous microarray analysis (Marin et al., 2004), we analyzed similar time points after salt shock: 0, 0.5, 2 and $24 \mathrm{~h}$. Labeling and hybridization were performed as described (Klähn et al., 2015). Three $\mu \mathrm{g}$ of RNA were used for the labeling reaction and $1.65 \mu \mathrm{g}$ of labeled RNA for the hybridization. The microarray hybridization was performed in duplicates for each sampling point. Almost all features on the microarray chip were covered by several independent probes. In addition, it contained technical replicates for each single probe. Hence, mean values for all probes of a given feature were used for the final calculation of fold changes. Data processing and statistical evaluation was performed using the R software as described (Klähn et al., 2015). The full dataset is accessible from the GEO database with the accession number GSE174316 (accession for Reviewers via: izupwswenzelfkv).

In Suppl. Table S1 transcripts are separated into mRNAs, asRNAs, other ncRNAs, 5'UTRs and transcripts derived from internal TSSs, i.e. within CDS (int). However, it should be noted that for every category overlaps are possible, i.e. the annotation as well as microarray detection of ncRNAs is often ambiguous since they can overlap with UTRs. Thus, all features labeled with the systematic term "NC-\#" are referred as "potentially" trans-acting ncRNAs. For the evaluation presented in the text only ncRNAs with an annotation based on Mitschke et al. (2011) were considered.

\section{Cluster analysis}

Clustering analysis for the microarray time series was performed using the mfuzz R package (Kumar and Futschik, 2007). To reduce noise and false positives, only 3831 transcripts entered the clustering analysis that had a difference in their absolute expression values higher than $\log _{2} \geq|1|$ in at least one of these comparisons. The optimal cluster number was determined with the Elbow method using the minimum centroid distance and an estimated "fuzzifier" parameter of $m=2.53$, yielding four clusters. Transcripts with low membership values $<0.5$ were removed. However, those transcripts were still included if the combined membership values for two similar clusters (e.g. cluster $1+2$ and $3+4$ ) exceeded $>0.6$. 


\section{Proteomic methods}

819 Cells from four biological replicates of salt-acclimated and control cultures, respectively, were

820 broken with glass beads using Precellys 24 homogenizer (peqLab Biotechnologie $\mathrm{GmbH}$, 821 Erlangen, Germany) in non-denaturing buffer containing $10 \mathrm{mM}$ Tris/ $\mathrm{HCl}, \mathrm{pH}$ 7.4, $138 \mathrm{mM}$ $822 \mathrm{NaCl}, 2.7 \mathrm{mM} \mathrm{KCl}, 1 \mathrm{mM} \mathrm{MgCl}$. After withdrawing an aliquot of each cell extract as total 823 protein the remaining cell extracts were fractionated into debris, soluble, and membrane824 enriched fractions. Protein samples were reduced with dithiothreitol, alkylated with 825 iodoacetamide and digested with trypsin in sodium deoxycholate-containing buffer solution 826 (Pappesch et al. 2017).

827 LC-HDMS ${ }^{\mathrm{E}}$ analyses of desalted peptide samples supplemented with $40 \mathrm{fmol}$ of Hi3 Phos B 828 standard for protein absolute quantification (Waters) were carried out using a nanoAcquity 829 UPLC system (Waters) coupled to a Waters Synapt G2-S mass spectrometer (Pade et al., 830 2017). The Synapt G2-S instrument was operated in data-independent mode with ion831 mobility separation as an additional dimension of separation (referred to as $\mathrm{HDMS}^{\mathrm{E}}$ ). Single measurements of the four biological replicates of the total extract, the soluble fraction and the 833 membrane-enriched fraction were carried out, while pooled samples of the debris fraction 834 were measured in triplicate.

835 Progenesis QI for Proteomics version 4.1 (Nonlinear Dynamics, Newcastle upon Tyne, UK) 836 was used for raw data processing, protein identification and label free quantification. Proteins 837 were quantified by the absolute quantification $\mathrm{Hi3}$ method using $\mathrm{Hi3}$ Phos B Standard 838 (Waters) as reference (Silva et al. 2006). Results were given as fmol on column. Proteins identified in one fraction by at least two unique peptides were included in the quantitative analysis. Protein abundance changes between salt-acclimated and control cells by a factor of at least 1.5 , accompanied by ANOVA $p$-values $<0.05$ were regarded as significant. To determine a combined fold change value for the total extract and the three subcellular fractions, first a weighted fold change value for the three subcellular fractions was calculated by summing up the corresponding protein amounts (measured as fmol on column) and dividing the value from salt-acclimated cells by the value from controls. Since the debris fraction contained only a minor part of about $10 \%$ of the total protein, the protein amounts of the debris fraction were divided by ten. In a second step, the final combined fold change value was calculated as the average of the weighted fold change from the subcellular fractions and the fold change of the total extract.

850 A detailed description of the proteome method can be found in the Supplementary Material.

851 The mass spectrometry proteomics data have been deposited to the ProteomeXchange 852 Consortium via the PRIDE (Vizcaíno et al., 2013) partner repository with the dataset identifier 853 PXD026118 and 10.6019/PXD026118. Data are available via ProteomeXchange with 
854 identifier PXD026118 (Reviewer account details: Username:

855 reviewer_pxd026118@ebi.ac.uk; Password: NjhSNM7Z).

\section{Metabolomic methods}

857 Low molecular mass compounds were extracted from cells with ethanol (80\%, HPLC grade, 858 Roth, Germany) at $65^{\circ} \mathrm{C}$ for $2 \mathrm{~h}$. The soluble sugars were analyzed by gas-liquid 859 chromatography using a defined amount of sorbitol as internal standard as described by 860 Hagemann et al. (2008). Other metabolites were analyzed by LC-MS/MS using the high861 performance liquid chromatograph mass spectrometer system LCMS-8050 (Shimadzu, 862 Japan). One microgram of carnitine was added per sample as internal standard for LC$863 \mathrm{MS} / \mathrm{MS}$ analyses. The dry extracts were dissolved in $200 \mu \mathrm{l}$ MS-grade water and filtered 864 through $0.2 \mu \mathrm{m}$ filters (Omnifix $®-F$, Braun, Germany). The cleared supernatants were 865 separated on a pentafluorophenylpropyl (PFPP) column (Supelco Discovery HS FS, $3 \mu \mathrm{m}$, $866150 \times 2.1 \mathrm{~mm}$ ) with a mobile phase containing $0.1 \%$ formic acid. The compounds were eluted 867 at a rate of $0.25 \mathrm{ml} \mathrm{min}^{-1}$ using the following gradient: 1 min $0.1 \%$ formic acid, $95 \%$ water, $5 \%$ 868 acetonitrile, within 15 min linear gradient to $0.1 \%$ formic acid, 5\% distilled water, 95\% 869 acetonitrile, $10 \mathrm{~min}$ 0.1\% formic acid, 5\% distilled water, 95\% acetonitrile. Aliquots were 870 continuously injected in the MS/MS part and ionized via electrospray ionization (ESI). The 871 compounds were identified and quantified using the multiple reaction monitoring (MRM) 872 values given in the LC-MS/MS method package and the LabSolutions software package 873 (Shimadzu, Japan). The metabolites were determined as relative metabolite abundances 874 (fold changes), which were calculated after normalization of signal intensity to that of the 875 internal standard carnitine.

\section{ACKNOWLEDGEMENTS}

877 The technical assistance of Klaudia Michl (University of Rostock) is acknowledged.

\section{FUNDING INFORMATION}

879 Funded by the German research Foundation (DFG) - "SCyCode" research group FOR 2816 880 (DFG ID 397695561) to MH and WRH, individual grant KL 3114/2-1 to SK, and the Research 881 Training Group "MelnBio" GRK2344 (DFG ID 322977937) to MR. The LC-MS/MS equipment 882 at University of Rostock was financed through the HBFG program (GZ: INST 264/125-1 883 FUGG). 


\section{AUTHOR CONTRIBUTION}

$\mathrm{MH}$ designed the study. SK, MR, JG \& WRH performed and evaluated the transcriptomic analyses. SM performed and evaluated the proteome experiments. $\mathrm{MH}$ performed and evaluated the metabolome analyses. $\mathrm{MH}, \mathrm{WRH}, \mathrm{SK} \& \mathrm{SM}$ wrote the manuscript.

\section{CONFLICT Of INTEREST}

The authors declare no conflicts of interest.

\section{References}

Aikawa S, Nishida A, Hasunuma T, Chang JS, Kondo A (2019) Short-term temporal metabolic behavior in halophilic cyanobacterium Synechococcus sp. strain PCC 7002 after salt shock. Metabolites 9: 297

Azad AK, Sato R, Ohtani K, Sawa Y, Ishikawa T, Shibata H (2011) Functional characterization and hyperosmotic regulation of aquaporin in Synechocystis sp. PCC 6803. Plant Sci 180: 375-382

Beuf L, Bédu S, Durand MC, Joset F (1994) A protein involved in co-ordinated regulation of inorganic carbon and glucose metabolism in the facultative photoautotrophic cyanobacterium Synechocystis PCC6803. Plant Mol Biol 25: 855-864

Billis K, Billini M, Tripp HJ, Kyrpides NC, Mavromatis K. 2014. Comparative transcriptomics between Synechococcus PCC 7942 and Synechocystis PCC 6803 provide insights into mechanisms of stress acclimation. PLoS One 9: e109738.

Bolay P, Muro-Pastor MI, Francisco J Florencio FJ, Klähn S (2018) The distinctive regulation of cyanobacterial glutamine synthetase. Life 8: 52

Cheung KJ, Badarinarayana V, Selinger DW, Janse D, Church GM (2003) A microarraybased antibiotic screen identifies a regulatory role for supercoiling in the osmotic stress response of Escherichia coli. Genome Res 13: 206-215

Chisti Y (2013) Constraints to commercialization of algal fuels. J Biotech 167: 201-214

Clarkson J, Campbell ID, Yudkin MD (2004) Efficient regulation of sigmaF, the first sporulation-specific sigma factor in B. subtilis. J Mol Biol 342: 1187-1195

Cui J, Sun T, Chen L, Zhang W (2020) Engineering salt tolerance of photosynthetic cyanobacteria for seawater utilization. Biotechnol Adv 43: 107578 
Cui J, Sun T, Chen L, Zhang W (2021) Salt-tolerant Synechococcus elongatus UTEX 2973 obtained via engineering of heterologous synthesis of compatible solute glucosylglycerol. Front Microbiol 12: 650217

Dorman CJ, Dorman MJ (2016) DNA supercoiling is a fundamental regulatory principle in the control of bacterial gene expression. Biophys Rev 8: 209-220

Dühring U, Axmann IM, Hess WR, Wilde A (2006) An internal antisense RNA regulates expression of the photosynthesis gene isiA. Proc Natl Acad Sci USA 103: 7054-7058

Eisenhut M, Georg J, Klähn S, Sakurai I, Mustila H, Zhang P, Hess WR, Aro EM (2012) The antisense RNA As1_flv4 in the cyanobacterium Synechocystis sp. PCC 6803 prevents premature expression of the flv4-2 operon upon shift in inorganic carbon supply. J Biol Chem 287: 33153-33162

Elanskaya IV, Karandashova IV, Bogachev AV, Hagemann M (2002) Functional analysis of the $\mathrm{Na}^{+} / \mathrm{H}^{+}$antiporter encoding genes of the cyanobacterium Synechocystis PCC 6803. Biochemistry (Moscow) 67: 432-440

Fulda S, Mikkat S, Huang F, Huckauf J, Marin K, Norling B, Hagemann M (2006) Proteome analysis of salt stress response in the cyanobacterium Synechocystis $\mathrm{sp}$. strain PCC 6803. Proteomics 6: $2733-2745$

Galmozzi CV, Florencio FJ, Muro-Pastor MI (2016) The cyanobacterial ribosomal-associated protein LrtA is involved in post-stress survival in Synechocystis sp PCC 6803. PLoS One 11: e0159346

Gao L, Wang J, Ge H, Fang L, Zhang Y, Huang X, Wang Y (2015a)Toward the complete proteome of Synechocystis sp. PCC 6803. Photosynth Res 126: 203-219

Gao L, Ge H, Huang X, Liu K, Zhang Y, Xu W, Wang Y (2015b) Systematically ranking the tightness of membrane association for peripheral membrane proteins (PMPs). Mol Cell Proteomics 14: 340-353

Gao F, Zhao J, Chen L, Battchikova N, Ran Z, Aro EM, Ogawa T, Ma W (2016) The NDH1L-PSI supercomplex Is important for efficient cyclic electron transport in cyanobacteria. Plant Phys 172: 1451-1464

Georg J, Dienst D, Schürgers N, Wallner T, Kopp D, Stazic D, et al. (2014) The small regulatory RNA SyR1/PsrR1 controls photosynthetic functions in cyanobacteria. Plant Cell 26: 3661-3679

Georg J, Kostova G, Vurijoki L, Schön V, Kadowaki T, Huokko T, ez al. 2017. Acclimation of oxygenic photosynthesis to iron starvation is controlled by the SRNA IsaR1. Curr Biol 27: $1425-1436$ 
Georg J, Hess WR (2018) Wide-spread antisense transcription in prokaryotes. Microbiol Spectrum 6: RWR-0029-2018

Grigorieva G, Shestakov S. 1982. Transformation in the cyanobacterium Synechocystis sp. 6803. FEMS Microbiol Lett 13: 367-370

Gärtner K, Klähn S, Watanabe S, Mikkat S, Scholz I, Hess WR, Hagemann M (2019) Cytosine N4-methylation via M.Ssp6803II is involved in the regulation of transcription, fine-tuning of DNA replication and DNA repair in the cyanobacterium Synechocystis sp. PCC 6803. Front Microbiol 10: 1233

Hagemann M, Fulda S, Schubert H (1994) DNA, RNA and protein synthesis in the cyanobacterium Synechocystis sp. PCC 6803 adapted to different salt concentrations. Curr Microbiol 28: 201-207

Hagemann M, Schoor A, Jeanjean R, Zuther E, Joset F (1997) The stpA gene form Synechocystis sp. strain PCC 6803 encodes the glucosylglycerol-phosphate phosphatase involved in cyanobacterial osmotic response to salt shock. J Bacteriol 179: $1727-1733$

Hagemann M, Jeanjean R, Fulda S, Havaux M, Erdmann N (1999) Flavodoxin accumulation contributes to enhanced cyclic electron flow around photosystem I in salt-stressed cells of Synechocystis sp. PCC 6803. Physiol Plant 105: 670-678

Hagemann M, Ribbeck-Busch K, Klähn S, Hasse D, Steinbruch R, Berg G (2008) The plantassociated bacterium Stenotrophomonas rhizophila expresses a new enzyme for the synthesis of the compatible solute glucosylglycerol. J Bacteriol 190: 5898-5906

Hagemann M (2011) Molecular biology of cyanobacterial salt acclimation. FEMS Microbiol Rev 35: 87-123

Hagemann M, Hess WR (2018) Systems and synthetic biology for the biotechnological application of cyanobacteria. Curr Opin Biotech 49: 94-99

Hagemann M, Song S, Brouwer EM (2021) Inorganic carbon assimilation in cyanobacteria: Mechanisms, regulation, and engineering. In Hudson P, Lee SY, Nielsen J (eds.) Cyanobacteria Biotechnology, Wiley-Blackwell Biotechnology Series, Chapter 1, 1-31.

Hernandez-Prieto MA, Futschik ME (2012) CyanoEXpress: A web database for exploration and visualisation of the integrated transcriptome of cyanobacterium Synechocystis sp. PCC6803. Bioinformation 8: 634-638

Hein, S., Scholz, I., Voß, B., and Hess, W. R. (2013). Adaptation and modification of three CRISPR loci in two closely related cyanobacteria. RNA Biol 10: 852-864 
Huang F, Fulda S, Hagemann M, Norling B (2006) Proteomic screening of salt-stressinduced changes in plasma membranes of Synechocystis sp. strain PCC 6803. Proteomics 6: 910-920

lijima H, Nakaya Y, Kuwahara A, Hirai MY, Osanai T (2015) Seawater cultivation of freshwater cyanobacterium Synechocystis sp. PCC 6803 drastically alters amino acid composition and glycogen metabolism. Front Microbiol 6: 326

Jablonsky J, Papacek S, Hagemann M (2016) Different strategies of metabolic regulation in cyanobacteria: from transcriptional to biochemical control. Sci Rep 6: 33024

Kaneko T, Sato S, Kotani H, Tanaka A, Asamizu E, Nakamura Y, et al. (1996) Sequence analysis of the genome of the unicellular cyanobacterium Synechocystis sp. strain PCC6803. II. Sequence determination of the entire genome and assignment of potential protein-coding regions (supplement). DNA Res 3: 185-209

Kanesaki Y, Suzuki I, Allakhverdiev SI, Mikami K, Murata N (2002) Salt stress and hyperosmotic stress regulate the expression of different sets of genes in Synechocystis sp. PCC 6803. Biochem Biophys Res Commun 290: 339-348

Khan RI, Wang Y, Afrin S, Wang B, Liu Y, Zhang X, Chen L, Zhang W, He L, Ma G (2016) Transcriptional regulator PrqR plays a negative role in glucose metabolism and oxidative stress acclimation in Synechocystis sp. PCC 6803. Sci Rep 6: 32507

Kizawa A, Kawahara A, Takashima K, Takimura Y, Nishiyama Y, Hihara Y (2017) The LexA transcription factor regulates fatty acid biosynthetic genes in the cyanobacterium Synechocystis sp. PCC 6803. Plant J 92: 189-198

Klähn S, Steglich C, Hess WR, Hagemann M (2010a) Glucosylglycerate: a secondary compatible solute common to marine cyanobacteria from nitrogen-poor environments. Environ Microbiol 12: 83-94

Klähn S, Höhne A, Simon E, Hagemann M (2010b) The gene ss/3076 encodes a protein mediating the salt-induced expression of ggpS for the biosynthesis of the compatible solute glucosylglycerol in Synechocystis sp. strain PCC 6803. J Bacteriol 192: 44034412

Klähn S, Schaal C, Georg J, Baumgartner D, Knippen G, Hagemann M, et al. (2015) The SRNA NsiR4 is involved in nitrogen assimilation control in cyanobacteria by targeting glutamine synthetase inactivating factor IF7. Proc Natl Acad Sci USA 112: E6243E6252 
Kirsch F, Pade N, Klähn S, Hess WR, Hagemann M (2017) The glucosylglycerol degrading enzyme GghA is involved in the acclimation to fluctuating salinities of the cyanobacterium Synechocystis sp. strain PCC 6803. Microbiology 163: 1319-1328

Kirsch F, Klähn S, Hagemann M (2019) Salt-regulated accumulation of the compatible solutes sucrose and glucosylglycerol in cyanobacteria and its biotechnological relevance. Front Microbiol 10: 2139

Koksharova O, Schubert M, Shestakov S, Cerff R (1998) Genetic and biochemical evidence for distinct key functions of two highly divergent GAPDH genes in catabolic and anabolic carbon flow of the cyanobacterium Synechocystis sp. PCC 6803. Plant Mol Biol 36: 183-194

Kondo K, Geng XX, Katayama M, Ikeuch M (2005). Distinct roles of CpcG1 and CpcG2 in phycobilisome assembly in the cyanobacterium Synechocystis sp. PCC 6803. Photosynth Res 84: 269-273

Kopf M, Klähn S, Scholz I, Matthiessen JK, Hess WR, Voß B (2014) Comparative analysis of the primary transcriptome of Synechocystis sp. PCC 6803. DNA Res 21: 527-539

Kopf M, Hess WR (2015) Regulatory RNAs in photosynthetic cyanobacteria. FEMS Microbiol Rev 39: 301-315

Kumar L, Futschik ME (2007) Mfuzz: a software package for soft clustering of microarray data. Bioinformation 2: 5-7

Lei H, Chen G, Wang Y, Ding Q, Wei D (2014) Sll0528, a site-2-protease, is critically involved in cold, salt and hyperosmotic stress acclimation of cyanobacterium Synechocystis sp PCC 6803. Int J Mol Sci 15: 22678-22693

Levina N, Totemeyer S, Stokes NR, Louis P, Jones MA, Booth IR (1999) Protection of Escherichia coli cells against extreme turgor by activation of MscS and MscL mechanosensitive channels: identification of genes required for MscS activity. EMBO J 18: $1730-1737$

Li H, Singh AK, McIntyre LM, Sherman LA (2004) Differential gene expression in response to hydrogen peroxide and the putative PerR regulon of Synechocystis sp. strain PCC 6803. J Bacteriol 186: 3331-3345

Liu Y, Beyer A, Aebersold R (2016) On the dependency of cellular protein levels on mRNA abundance. Cell 165: 535-550

Liu X, Miao R, LindbergP, Lindblad P (2019a) Modular engineering for efficient photosyntheteic biosynthesis of 1-butanol from $\mathrm{CO}_{2}$ in cyanobacteria. Energy Environ Sci 12: 2765-2777 
Liu H, Weis, DA, Zhang MM, Cheng M, Zhang B, Zhang H, Gerstenecker GS, Pakrasi HB, Gross ML, Blankenship RE (2019b) Phycobilisomes harbor FNR L in cyanobacteria. mBio 10: e00669-19

Marin K, Zuther E, Kerstan T, Kunert A, Hagemann M (1998) The ggpS gene from Synechocystis sp. strain PCC 6803 encoding glucosyl-glycerol-phosphate synthase is involved in osmolyte synthesis. J Bacteriol 180: 4843-4849

Marin K, Huckauf J, Fulda S, Hagemann M (2002) Salt-dependent expression of glucosylglycerol-phosphate synthase, involved in osmolyte synthesis in the cyanobacterium Synechocystis sp. strain PCC 6803. J Bacteriol 184: 2870-2877

Marin K, Suzuki I, Yamaguchi K, Ribbeck K, Yamamoto H, Kanesaki Y, Hagemann M, Murata N (2003) Identification of histidine kinases that act as sensors in the perception of salt stress in Synechocystis sp. PCC 6803. Proc Natl Acad Sci USA 100: $9061-9066$

Marin K, Kanesaki Y, Los DA, Murata N, Suzuki I, Hagemann M (2004) Gene expression profiling reflects physiological processes in salt acclimation of Synechocystis sp. strain PCC 6803. Plant Physiol 136: 3290-3300

Matsuhashi A, Tahara H, Ito Y, Uchiyama J, Ogawa S, Ohta H (2015) Slr2019, lipid A transporter homolog, is essential for acidic tolerance in Synechocystis sp PCC6803. Photosynth Res 125: 267-277

Mikkat S, Hagemann M (2000) Molecular analysis of the ggtBCD operon of Synechocystis sp. strain PCC 6803 encoding the substrate-binding protein and the transmembrane proteins of an ABC transporter. Arch Microbiol 174: 273-282

Mikkat S, Milkowski C, Hagemann M (2000) The gene s/l0273 of the cyanobacterium Synechocystis sp. strain PCC 6803 encodes a protein essential for growth at low $\mathrm{Na}^{+} / \mathrm{K}^{+}$ratios. Plant Cell Environm 23: 549-559

Mitschke J, Georg J, Scholz I, Sharma CM, Dienst D, Bantscheff J, et al. (2011) An experimentally anchored map of transcriptional start sites in the model cyanobacterium Synechocystis sp. PCC6803. Proc Natl Acad Sci USA 108: 21242129

Novak JF, Stirnberg M, Roenneke B, Marin K (2011) A novel mechanism of osmosensing, a salt-dependent protein-nucleic acid interaction in the cyanobacterium Synechocystis Species PCC 6803. J Biol Chem 286: 3235-3241 
1081

1082

1083

1084

1085

1086

1087

1088

1089

1090

1091

1092

1093

1094

1095

1096

1097

1098

1099

1100

1101

1102

1103

1104

1105

1106

1107

1108

1109

1110

1111

Nowaczyk MM, Krause K, Mieseler M, Sczibilanski A, Ikeuchi M, Rögner M (2012) Deletion of psbJ leads to accumulation of Psb27-Psb28 photosystem II complexes in Thermosynechococcus elongatus. Biochim Biophys Acta 1817: 1339-1345

Pade N, Compaoré J, Klähn S, Stal LJ, Hagemann M (2012) The marine cyanobacterium Crocosphaera watsonii WH8501 synthesizes the compatible solute trehalose by a laterally acquired OtsAB fusion protein. Environ Microbiol 14: 1261-1271

Pade N, Hagemann M (2014) Salt acclimation of cyanobacteria and their application in biotechnology. Life 5: 25-49

Pade N, Michalik D, Ruth W, Belkin N, Hess WR, Berman-Frank I, Hagemann M (2016) Trimethylated homoserine functions as the major compatible solute in the globally significant oceanic cyanobacterium Trichodesmium. Proc Natl Acad Sci USA 113: 13191-13196

Pade N, Mikkat S, Hagemann M (2017) Ethanol, glycogen and glucosylglycerol represent competing carbon pools in ethanol-producing cells of Synechocystis sp. PCC 6803 under high-salt conditions. Microbiology 163: 300-307

Pappesch R, Warnke P, Mikkat S, Normann J, Wisniewska-Kucper A, Huschka F, Wittmann M, Khani A, Schwengers O, Oehmcke-Hecht S, Hain T, Kreikemeyer B, Patenge N. (2017) The regulatory small RNA marS supports virulence of Streptococcus pyogenes. Sci Rep 7: 12241

Pattanayak GK, Liao Y, Wallace EWJ, Budnik B, Drummond DA, Rust MJ (2020) Daily cycles of reversible protein condensation in cyanobacteria. Cell Rep 32: 108032

Pereira SB, Santos M, Leite JP, Flores C, Eisfeld C, Büttel Z, Mota R, Rossi F, De Philippis R, Gales L, Morais-Cabral JH, Tamagnini P (2019) The role of the tyrosine kinase Wzc (Sll0923) and the phosphatase Wzb (Slr0328) in the production of extracellular polymeric substances (EPS) by Synechocystis PCC 6803. Microbiol open 8: e00753

Perozo E, Kloda A, Cortes DM, Martinac B. (2001) Site-directed spin-labeling analysis of reconstituted MscL in the closed state. J Gen Physiol 118: 193-206

Pinto FL, Thapper A, Sontheim W, Lindblad P (2009) Analysis of current and alternative phenol based RNA extraction methodologies for cyanobacteria. BMC Mol Biol 10: 79

Prakash JS, Sinetova M, Zorina A, Kupriyanova E, Suzuki I, Murata N, Los DA (2009) DNA supercoiling regulates the stress-inducible expression of genes in the cyanobacterium Synechocystis. Mol Biosyst 5: 1904-1912

Qiao J, Huang S, Te R, Wang J, Chen L, Zhang W (2013) Integrated proteomic and transcriptomic analysis reveals novel genes and regulatory mechanisms involved in 
salt stress responses in Synechocystis sp. PCC 6803. Appl Microbiol Biotechnol 97: 8253-8264

1114

1115

1116

1117

1118

1119

1120

1121

1122

1123

1124

1125

1126

1127

1128

1129

1130

1131

1132

1133

1134

1135

1136

1137

1138

1139

1140

1141

1142

1143

1144

Qiao Y, Wang W, Lu X (2020) Engineering cyanobacteria as cell factories for direct trehalose production from $\mathrm{CO}_{2}$. Metab Eng 62: 161-171

Reed RH, Borowitzka LJ, Mackay MA, Chudek JA, FosterR, Warr SRC, et al. (1986) Organic solute accumulation in osmotically stressed cyanobacteria. FEMS Microbiol Lett 39: $51-56$

Reed RH, Stewart WDP (1985) Osmotic adjustment and organic solute accumulation in unicellular cyanobacteria from freshwater and marine habitats. Mar Biol 88: 1-9

Riediger M, Kadowaki T, Nagayama R, Georg J, Hihara Y, Hess WR (2019) Biocomputational analyses and experimental validation identify the regulon controlled by the redox-responsive transcription factor RpaB. iScience 15: 316-331

Riediger M, Spät P, Bilger R, Voigt K, Macek B, Hess WR (2021) Analysis of a photosynthetic cyanobacterium rich in internal membrane systems via gradient profiling by sequencing (Grad-seq). Plant Cell 33: 248-269

Ritter SPA, Lewis AC, Vincent SL, Lo LL, Cunha APA, Chamot D, Ensminger I, Espie GS, Owttrim GW (2020) Evidence for convergent sensing of multiple abiotic stresses in cyanobacteria. Biochim Biophys Acta Gen Subj 1864: 129462

Rübsam H, Kirsch F, Reimann V, Erban A, Kopka J, Hagemann M, Hess WR, Klähn S (2018) The iron-stress activated RNA 1 (IsaR1) coordinates osmotic acclimation and iron starvation responses in the cyanobacterium Synechocystis sp. PCC 6803. Environ Microbiol 20: 2757-2768

Sakurai I, Stazic D, Eisenhut M, Vuorio E, Steglich C, Hess WR, Aro EM (2012) Positive regulation of $p s b A$ gene expression by cis-encoded antisense RNAs in Synechocystis sp. PCC 6803. Plant Physiol 160: 1000-1010

Scholz I, Lange SJ, Hein S, Hess WR, Backofen R (2013) CRISPR-Cas systems in the cyanobacterium Synechocystis sp. PCC6803 exhibit distinct processing pathways involving at least two Cas6 and a Cmr2 protein. PLoS One 8: e56470

Schwarz D, Orf I, Kopka J, Hagemann M (2013) Recent applications of metabolomics toward cyanobacteria. Metabolites 3: 72-100

Shcolnick S, Shaked Y, Keren N (2007) A role for mrgA, a DPS family protein, in the internal transport of $\mathrm{Fe}$ in the cyanobacterium Synechocystis sp. PCC6803. Biochim Biophys Acta 1767: 814-819 
Shapiguzov A, Lyukevich AA, Allakhverdiev SI, Sergeyenko TV, Suzuki I, Murata N, Los DA (2005) Osmotic shrinkage of cells of Synechocystis sp. PCC 6803 by water efflux via aquaporins regulates osmostress-inducible gene expression. Microbiology 151: 447455

Shi L, Bischoff KM, Kennelly PJ (1999) The icfG gene cluster of Synechocystis sp. strain PCC 6803 encodes an Rsb/Spo-like protein kinase, protein phosphatase, and two phosphoproteins. J Bacteriol 181: 4761-4767

Shoumskaya MA, Paithoonrangsarid K, Kanesaki Y, Los DA, Zinchenko VV, Tanticharoen M, Suzuki I, Murata N (2005) Identical Hik-Rre systems are involved in perception and transduction of salt signals and hyperosmotic signals but regulate the expression of individual genes to different extents in Synechocystis. J Biol Chem 280: 21531-21538

Silva JC, Gorenstein MV, Li GZ, Vissers JPC, Geromanos SJ (2006) Absolute quantification of proteins by LCMSE: A virtue of parallel MS acquisition. Mol Cell Proteomics 5: 144-156

Spät P, Barske T, Maček B, Hagemann M (2021) Alterations in the $\mathrm{CO}_{2}$ availability induce alterations in the phospho-proteome of the cyanobacterium Synechocystis sp. PCC 6803. New Phytol 231: 1123-1137

Stanier RY, Kunisawa R, Mandel M, Cohen-Bazire G (1971) Purification and properties of unicellular blue-green algae (order Chroococcales). Bacteriol Rev 35: 171-205

Stokes NR, Murray HD, Subramaniam C, Gourse RL, Louis P, Bartlett W, Miller S, Booth IR (2003) A role for mechanosensitive channels in survival of stationary phase: regulation of channel expression by RpoS. Proc Natl Acad Sci USA 100: 1595915964

Summerfield TC, Sherman LA (2008) Global transcriptional response of the alkali-tolerant cyanobacterium Synechocystis sp. strain PCC 6803 to a pH 10 environment. Appl Environ Microbiol 74: 5276-5284

Takashima K, Nagao S, Kizawa A, Suzuki T, Dohmae N, Hihara Y (2020) The role of transcriptional repressor activity of LexA in salt-stress responses of the cyanobacterium Synechocystis sp. PCC 6803. Sci Rep 10: 17393

Toyoshima M, Tokumaru Y, Matsuda F, Shimizu H (2020) Assessment of protein content and phosphorylation level in Synechocystis sp. PCC 6803 under various growth conditions using quantitative phosphoproteomic analysis. Molecules 25: E3582 
Tyystjärvi T, Huokko T, Rantamäki S, Tyystjärvi E (2013) Impact of different group 2 sigma factors on light use efficiency and high salt stress in the cyanobacterium Synechocystis sp. PCC 6803. PLoS One 8: e63020

Uchiyama J, Asakura R, Moriyama A, Kubo Y, Shibata Y, Yoshino Y, Tahara H, Matsuhashi A, Sato S, Nakamura Y, Tabata S, Ohta H (2014) Sll0939 is induced by Slr0967 in the cyanobacterium Synechocystis sp. PCC6803 and is essential for growth under various stress conditions. Plant Physiol Biochem 81: 36-43

Vinnemeier J, Hagemann M (1999) Identification of salt-regulated genes in the genome of the cyanobacterium Synechocystis sp. strain PCC 6803 by subtractive RNA hybridization. Arch Microbiol 172: 377-386

Vizcaíno JA, Cote' RG, Csordas A, Dianes JA, Fabregat A, Foster JM, Griss J, Alpi E, Birim M, Contell J, et al. (2013) The Proteomics Identifications (PRIDE) database and associated tools: status in 2013. Nucl Acid Res 41: D1063-D1069

Wang HL, Postier BL, Burnap RL (2002) Polymerase chain reaction-based mutageneses identify key transporters belonging to multigene families involved in $\mathrm{Na}^{+}$and $\mathrm{pH}$ homeostasis of Synechocystis sp. PCC 6803. Mol Microbiol 44: 1493-1506

Wegener KM, Singh AK, Jacobs JM, Elvitigala T, Welsh EA, Keren N, Gritsenko MA, Ghosh BK, Camp DG 2nd, Smith RD, et al. (2010) Global proteomics reveal an atypical strategy for carbon/nitrogen assimilation by a cyanobacterium under diverse environmental perturbations. Mol Cell Proteomics 9: 2678-2689

Whitton BA, Potts M (2000) The ecology of cyanobacteria. Their diversity in time and space. Kluwer Academic Publishers, Dordrecht, The Netherlands

Wu W, Du W, Gallego RP, Hellingwerf KJ, van der Woude AD, Branco Dos Santos F (2020) Using osmotic stress to stabilize mannitol production in Synechocystis sp. PCC6803. Biotechnol Biofuels 13: 117

Zhan J, Steglich C, Scholz I, Hess WR, Kirilovsky D (2021) Inverse regulation of light harvesting and photoprotection mediated by a 3'end-derived SRNA in cyanobacteria. Plant Cell 33: 358-380

Zuther E, Schubert H, Hagemann M. 1998. Mutation of a gene encoding a putative glycoprotease leads to reduced salt tolerance, altered pigmentation, and cyanophycin accumulation in the cyanobacterium Synechocystis sp. strain PCC 6803. J Bacteriol 180: $1715-1722$ 


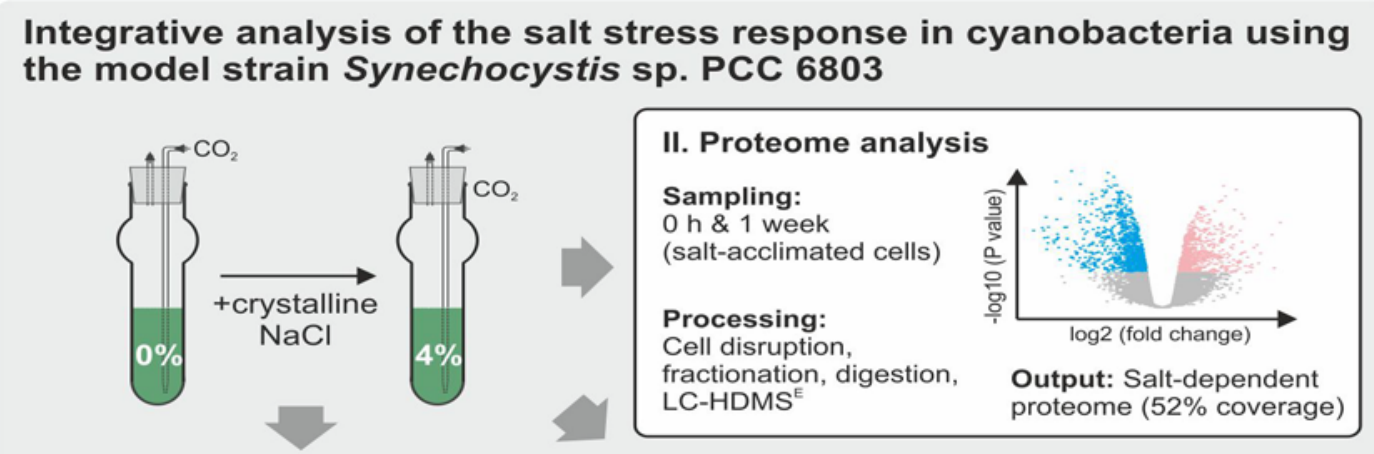

\section{Microarray analysis \\ Sampling: \\ $0,0.5,2 \& 24 \mathrm{~h}$ \\ Processing: \\ RNA extraction, direct labelling, \\ microarray hybridization}

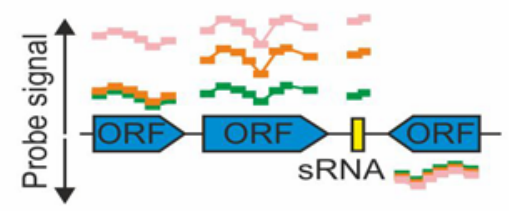

Output: Time-resolved, strand-specific transcript accumulation profile
III. Metabolome analysis

Sampling:

$0 \mathrm{~h} \& 1$ week (salt-acclimated cells)

Processing:

Ethanolic extraction, GC, LC-MS
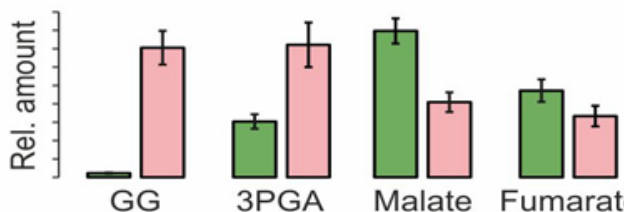

Malate Fumarate

Output: Salt-dependent metabolite patterns

1213 Figure 1. Overview on the applied approaches and conditions. Samples for microarray, 1214 proteome, and metabolome analyses were taken from cultures sparked with $\mathrm{CO}_{2}$-enriched air $(5 \%[v / v])$. Sampling points for each experiment are given in the panels. Further details about cultivation, salt treatment and sample processing are given in the Materials \& Methods section. 
(A)

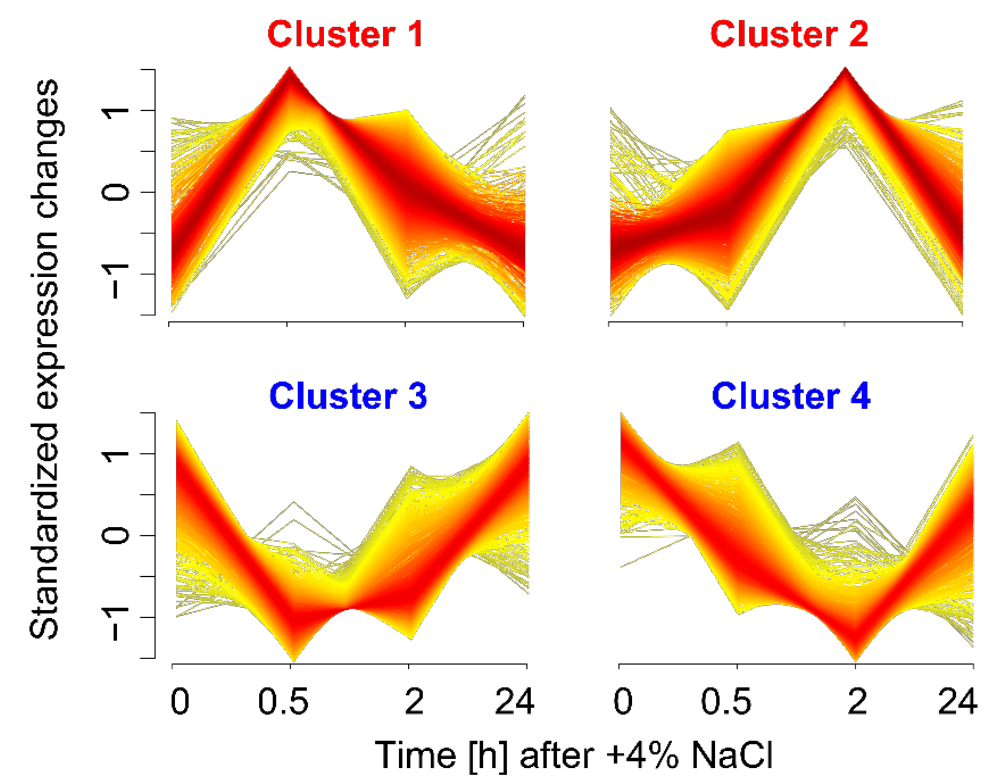

(B)
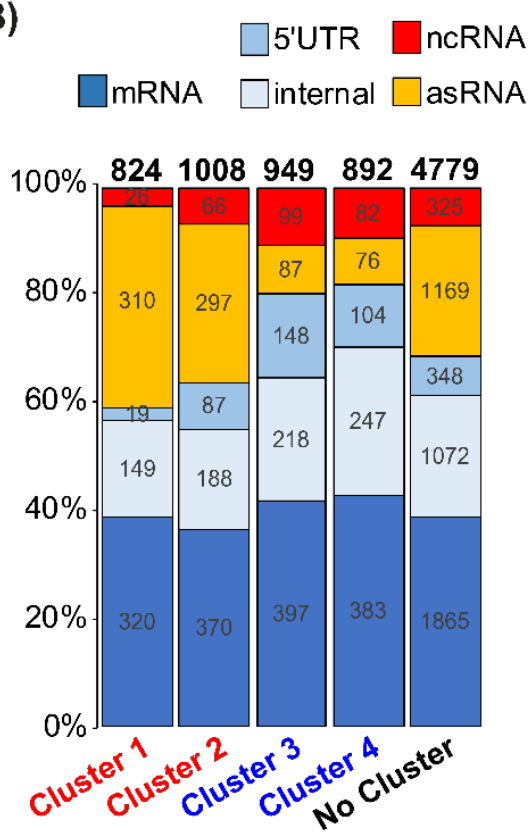

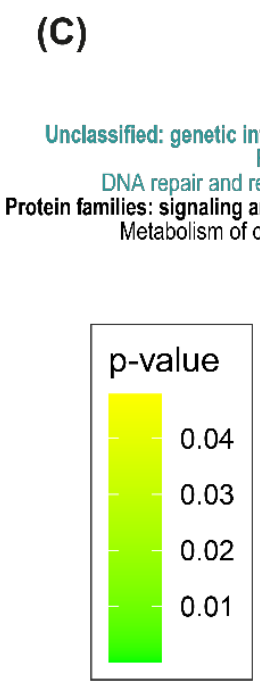

Figure 2. Cluster analysis of the salt stress microarray time series. A. Four major clusters of co-regulated transcripts were obtained. B. Distribution of different transcript types over the different clusters (see Suppl. Table S2 for the precise values and assignments). C: Functional enrichment analysis of proteins encoded by differentially regulated mRNAs according to KEGG Orthology (KO) terms for each cluster. Heatmap coloring represents the enrichment $p$-values $(y$ axis $=$ enriched $\mathrm{KO}$ terms, $\mathrm{x}$ axis $=$ genes, see Suppl. Table S3 for detailed information). 
(C) asRNA mRNA/CDS
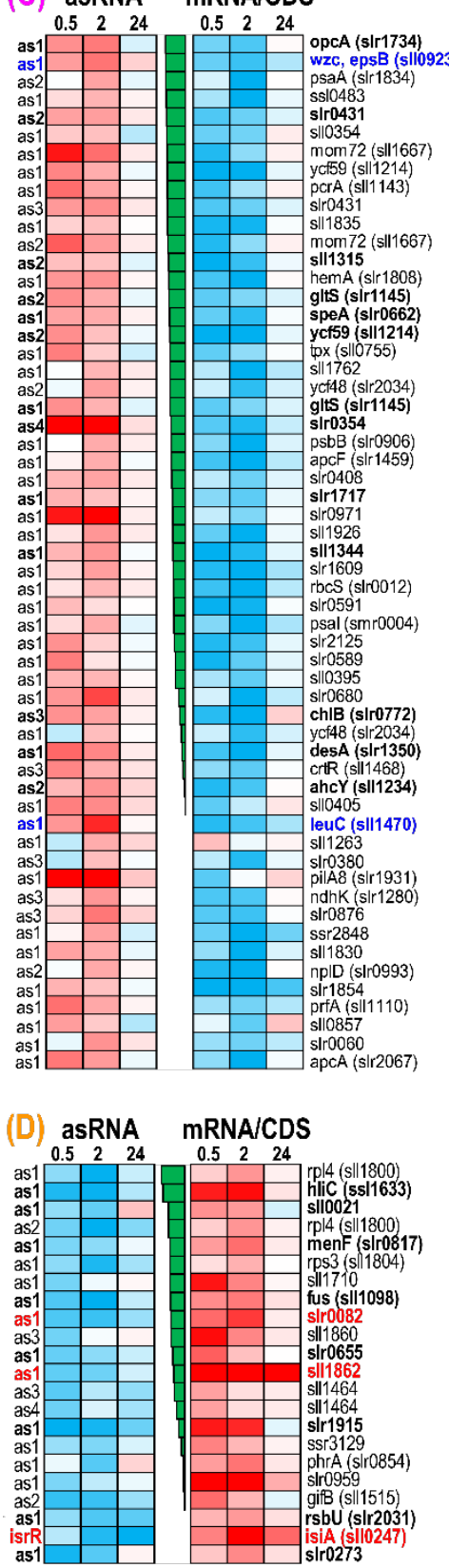
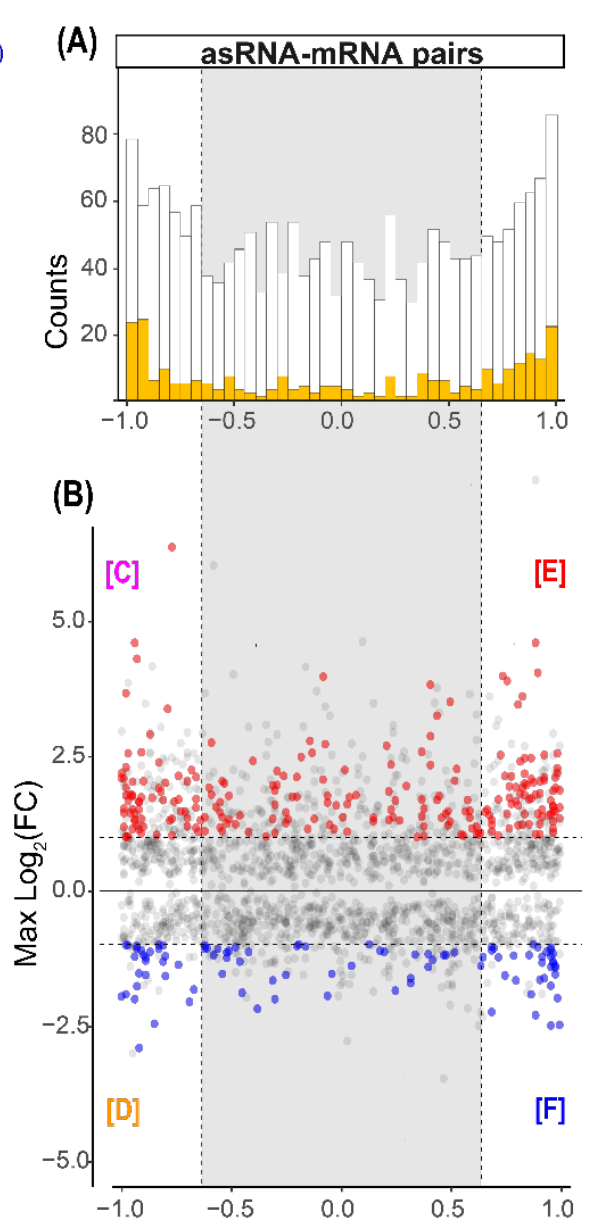

Pearson correlation coefficient to $\mathrm{mRNA} / \mathrm{CDS}$

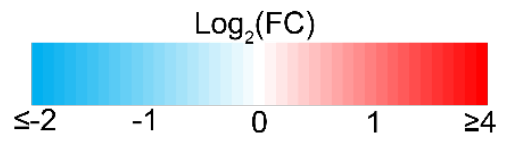

(E) asRNA mRNA/CDS

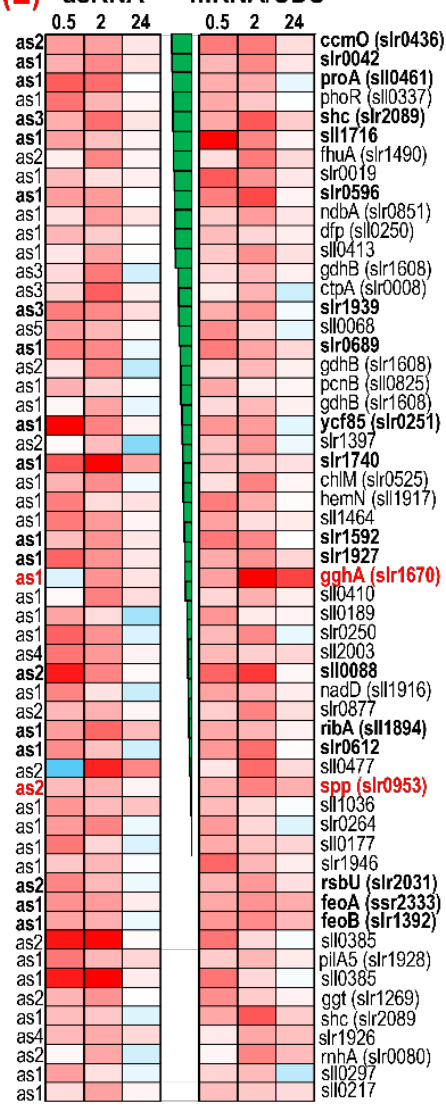

(F) asRNA mRNA/CDS

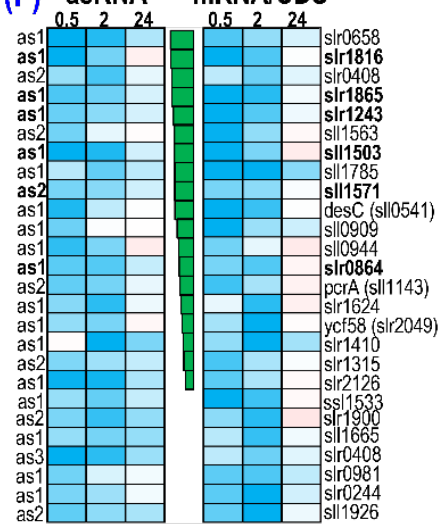

Figure 3. Comparison of salt-regulated mRNA:asRNA pairs with inverse or similar induction patterns. A. Histogram of Pearson correlation coefficients for expression profiles of all detected asRNA/mRNA pairs. Highlighted are asRNA/mRNA pairs, which both were assigned to an expression cluster with significant differential expression at least at one-time point. B. Scatterplot of maximum absolute $\log _{2}$ fold changes for every asRNA vs. Pearson correlation coefficients to its cognate mRNA. Colored points show the expression cluster assignment for the respective asRNA (red $=$ cluster $1+2$; blue $=$ cluster $3+4$ ). White backgrounds indicate strong correlation between asRNA/mRNA pairs (either $\geq 0.65$ or $\leq-$ 0.65). Numbers of individual cluster assignments for asRNA/mRNA pairs are indicated in the boxes. C. 57 pairs were asRNA-induced and mRNA-repressed, D. 22 pairs were asRNArepressed and mRNA-induced, E. 56 pairs were asRNA- and mRNA-induced, F. 26 pairs were asRNA- and mRNA-repressed. Only asRNA/mRNA pairs are given, which both were assigned to an expression cluster with a Pearson correlation coefficient of either $\geq 0.65$ or $\leq-$ 
0.65 at least at one-time point. The heat maps illustrate the $\log _{2}(\mathrm{FC})$ at the individual measurements from the microarray experiments for asRNAs (left) and mRNAs (right). The heatmaps are sorted according to Pearson correlation coefficient (center). Examples that are mentioned in the text are highlighted based on their expression. Bold font indicates at least two significant differential expression measurements per transcript. Details of the asRNA/mRNA analysis are given in Suppl. Table S4.

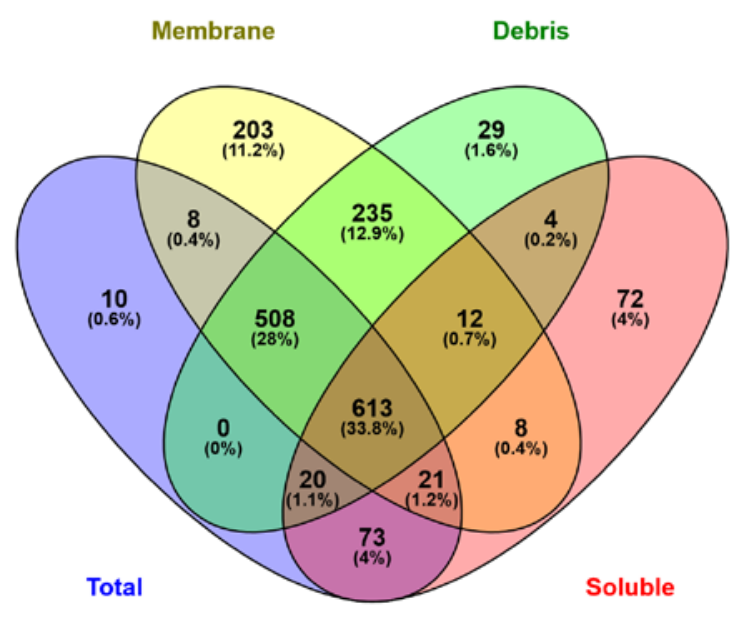

Figure 4. Overlap of the proteome among the different protein fractions. Venn diagram showing numbers and percentages of identified proteins in the total protein extract and in the subcellular fractions of debris, soluble or membrane proteins. 

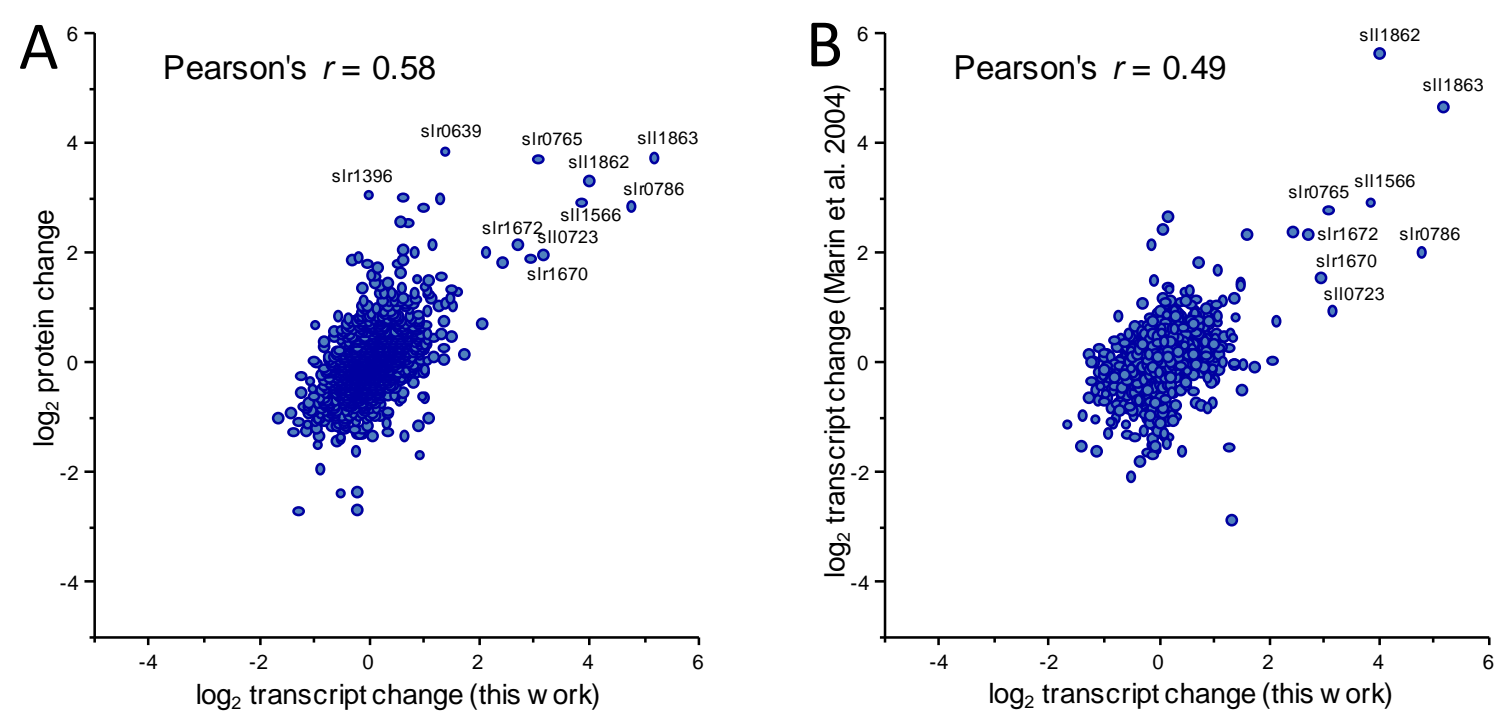

\begin{tabular}{|c|c|c|c|c|}
\hline $\begin{array}{c}\text { Pearson } \\
\text { correlation }\end{array}$ & Proteome & $\begin{array}{c}\text { Transcriptome } \\
\text { this work }\end{array}$ & $\begin{array}{c}\text { Transcriptome } \\
\text { Marin et al. 2004 }\end{array}$ & $\begin{array}{c}\text { Transcriptome } \\
\text { Billis et al. 2014 }\end{array}$ \\
\hline Proteome & 0.579 & 0.410 & 0.420 \\
\hline $\begin{array}{c}\text { Transcriptome } \\
\text { this work }\end{array}$ & 0.579 & 0.489 & 0.489 & 0.498 \\
\hline $\begin{array}{c}\text { Transcriptome } \\
\text { Marin et al. 2004 }\end{array}$ & 0.410 & 0.498 & 0.510 & 0.511 \\
\hline $\begin{array}{c}\text { Transcriptome } \\
\text { Billis et al. 2014 }\end{array}$ & 0.420 & & & \\
\hline
\end{tabular}

Figure 5. Correlation between transcriptome $(24 \mathrm{~h})$ and proteome $(7 \mathrm{~d})$ dynamics in obtained proteome data were compared with the previous transcriptomic study (Marin et al., 2004). Scatterplots display the correlation of protein and transcript ratios. 1749 transcripts with reported fold changes could be mapped to corresponding changes in protein abundances. Matches with ratio differences below $\log _{2} 1.5$-fold changes were considered to be similar. C. Table displaying Person's correlation coefficients between the different data sets. 

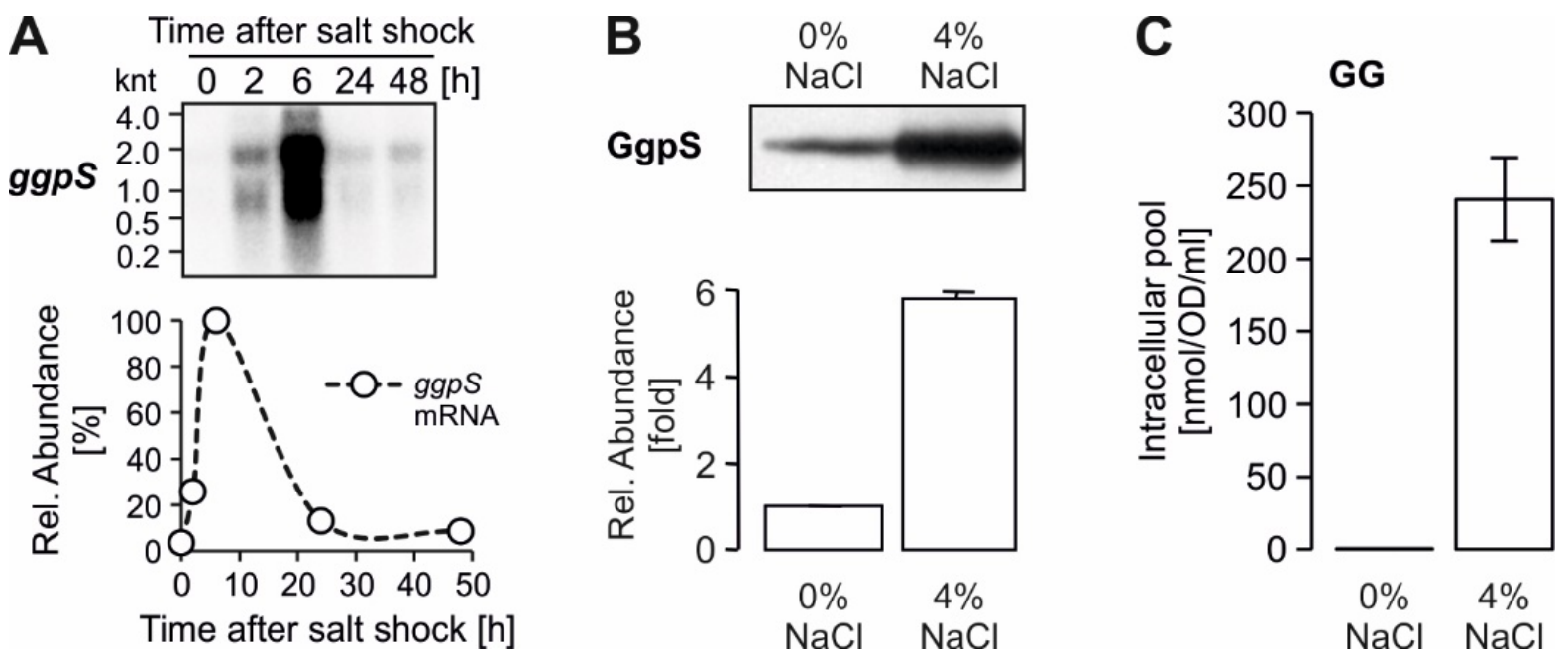

Figure 6. Salt-dependent up-regulation of the GG synthesis key enzyme, GGphosphate synthase (GgpS). A. Northern-blot showing accumulation kinetics of the ggpS transcript in response to salt shock of $4 \%[\mathrm{w} / \mathrm{v}] \mathrm{NaCl}$. Relative abundances were calculated after densitometric evaluation of the blot signals (signal obtained for $6 \mathrm{~h}$ was set as maximum, 100\%). B. Western blot confirming increased GgpS abundance in cells acclimated to $4 \% \mathrm{NaCl}$. Relative abundance was calculated after densitometric evaluation of blot signals (signal from control cells was set as 1 ). Data are the mean \pm SD of values obtained from three individual blots. C. Intracellular accumulation of GG in cells acclimated for 7 days to $4 \%$ $\mathrm{NaCl}$. Data are the mean \pm SD of 6 replicates. 


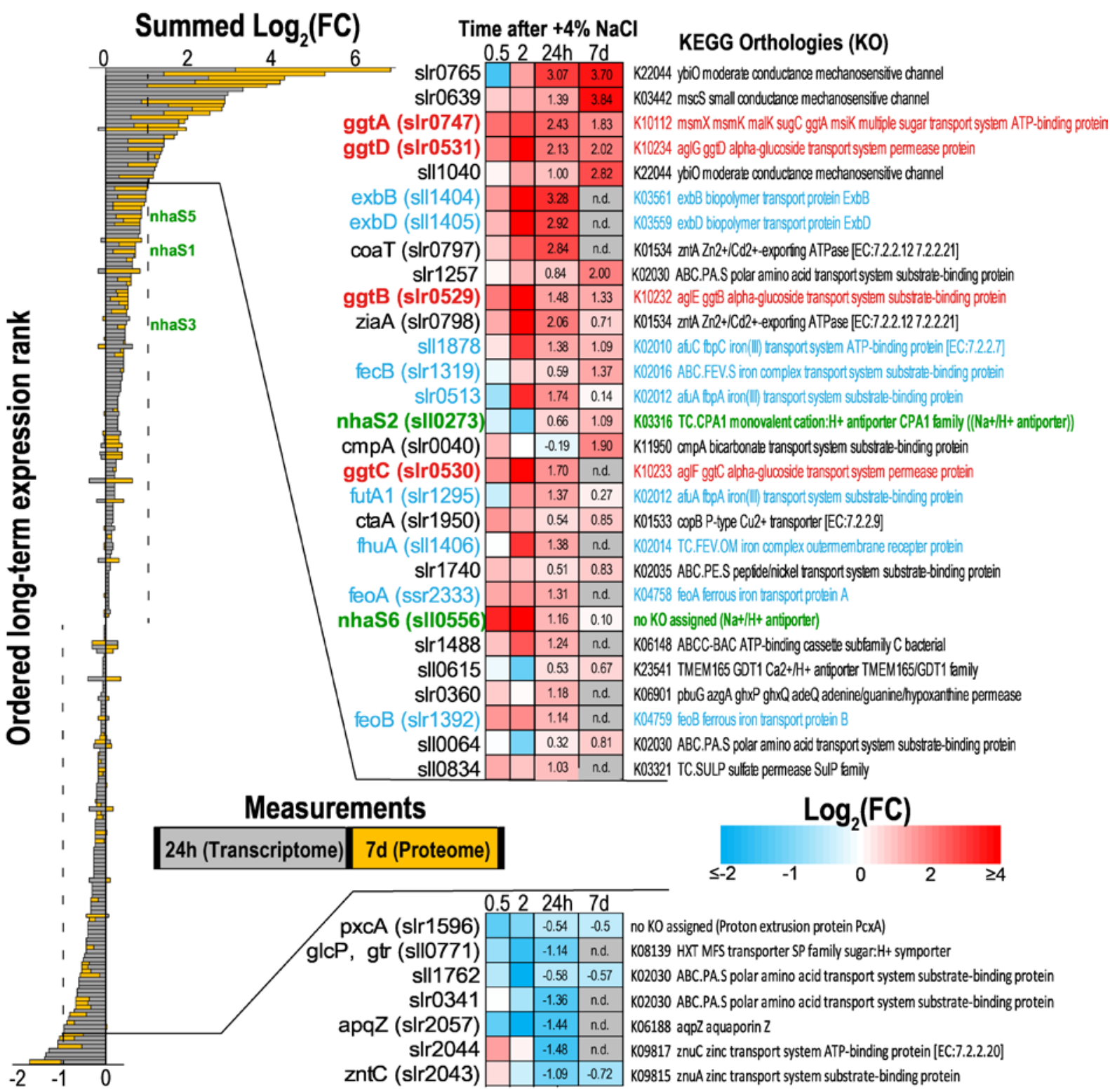

Figure 7. Ordered long-term expression ranks for transport-related genes. Ranks are ordered according to the summed $\log _{2}(F C)$ from the $24 \mathrm{~h}$ (transcriptome) and $7 \mathrm{~d}$ (proteome) measurements of salt-acclimated cells vs. control cells. The heatmaps on the right illustrate the $\log _{2}(\mathrm{FC})$ at the individual measurements from the microarray experiment $(0.5,2$, and $24 \mathrm{~h})$ and proteome measurements $(7 \mathrm{~d})$ for the top ranked transport related genes. Highlighted in Red = compatible solute transport $(g g t A B C D)$; Blue = related to iron transport, Green $=$ nhaS genes $\left(\mathrm{Na}^{+} / \mathrm{H}^{+}\right.$antiporter $)$. Detailed information is provided in Suppl. 


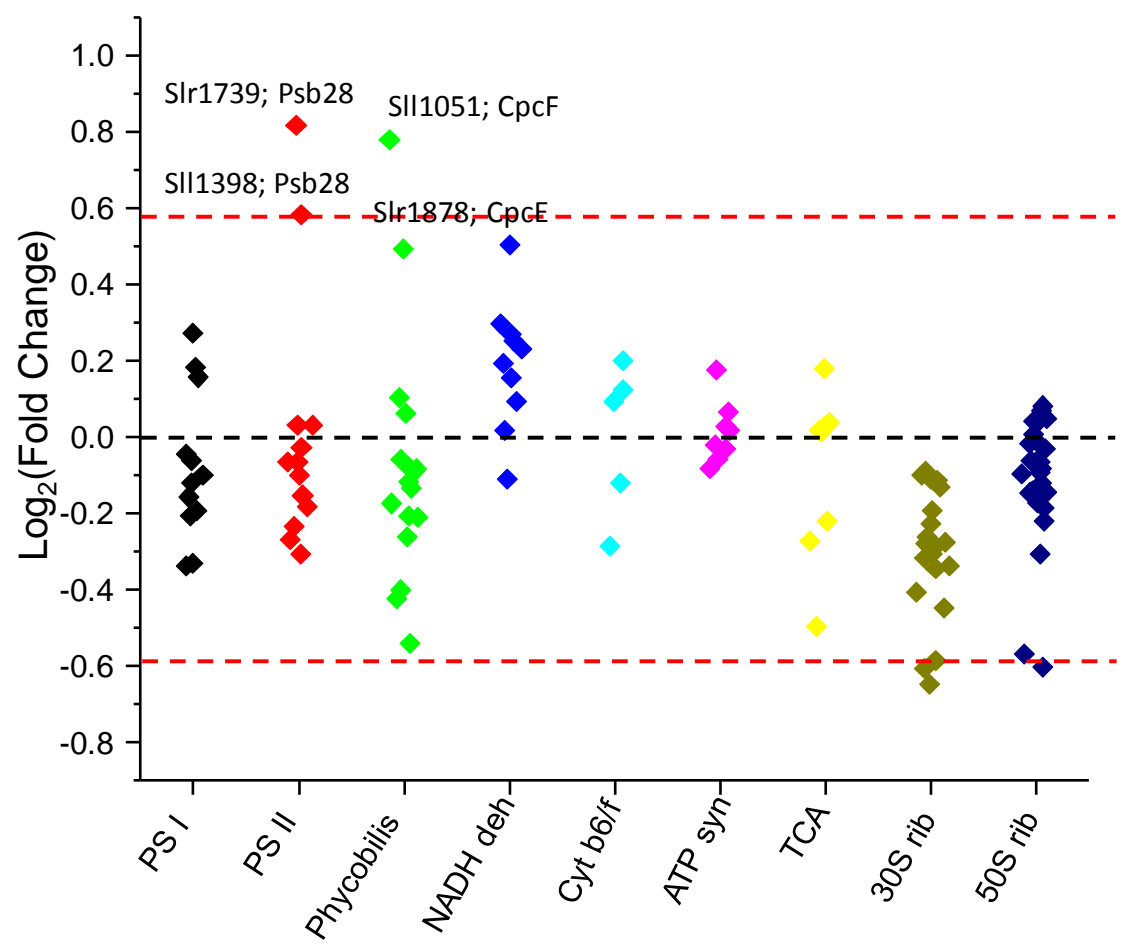

1292 Figure 8. Influence of the salinity on basic cellular processes. $\log _{2}$ fold change values (salt-acclimated/control) from the identified protein components of the indicated processes are plotted vertically aligned. 


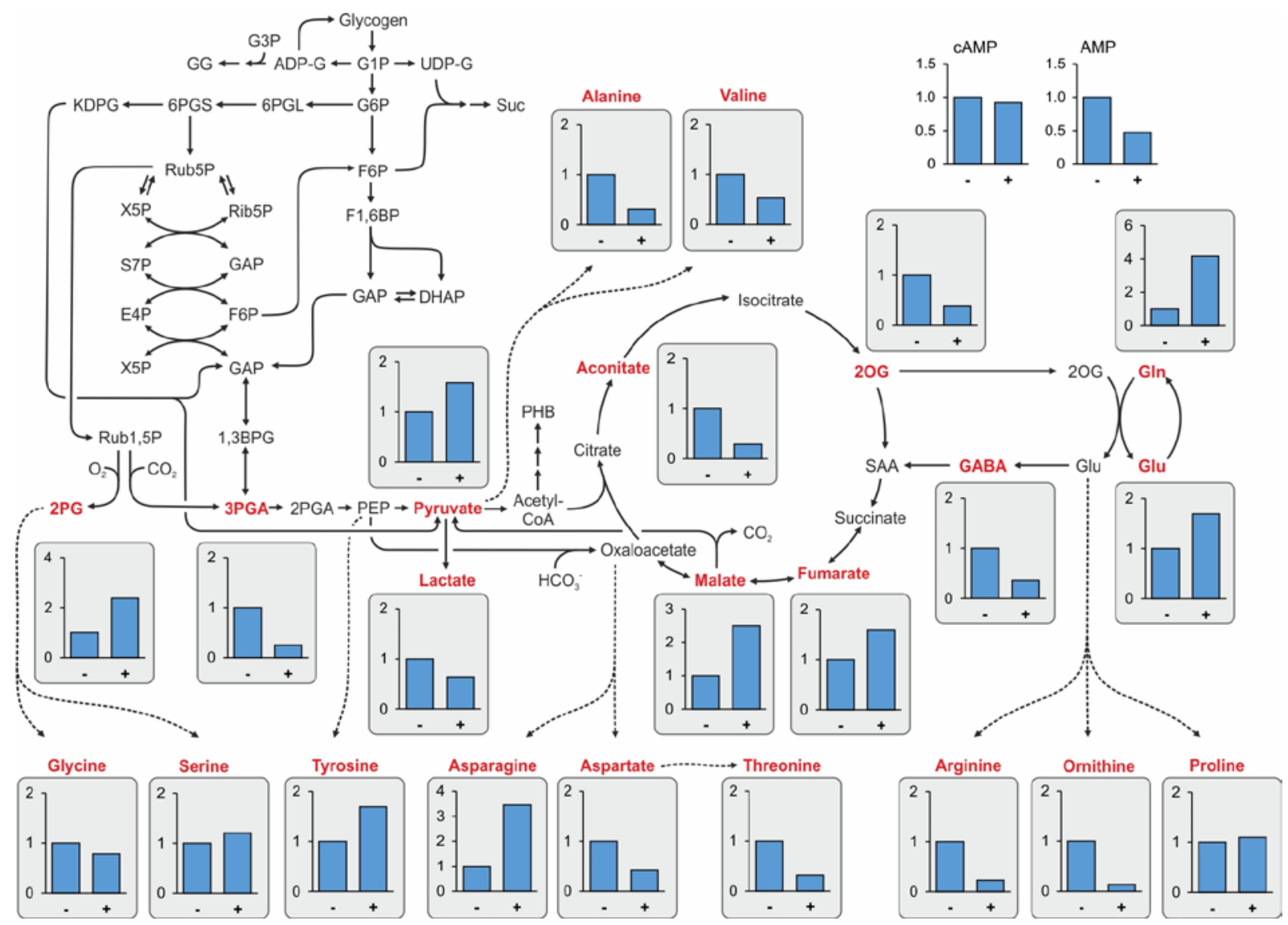

Figure 9. Alterations in the central carbon and nitrogen metabolism. Low molecular mass compounds were isolated from cells of Synechocystis 6803 grown in NaCl-free BG11 medium or medium supplemented with $4 \% \mathrm{NaCl}$ for 7 days. LC-MS/MS was used to estimate the relative levels ( $\mathrm{Y}$ axis show fold changes, amount in cells from $0 \% \mathrm{NaCl}$ cultivation (-) set to 1 and relative level at $4 \% \mathrm{NaCl}(+)$ is shown) of central metabolites as part of primary carbon and nitrogen metabolism. Shown are mean values from three biological replicates (details in Suppl. Table S10). Dotted arrows indicate that several enzymes are necessary to convert one metabolite into the other. The alteration in the compatible solute GG is shown in Fig. 6. 
Tables and Legends

Table 1. Global analysis of protein-coding genes showing altered transcript levels after salt shock of $684 \mathrm{mM} \mathrm{NaCl}$. A gene was regarded as induced or repressed if the $\log _{2}$ fold change was higher or lower than 1 or -1 ( $P$ value $<0.05$; n.a. - not analyzed).

\begin{tabular}{|l|c|c|c|c|}
\hline \multirow{4}{*}{ up-regulated mRNAs } & $\begin{array}{c}\text { Time after salt } \\
\text { shock }\end{array}$ & $\begin{array}{c}\text { Marin et al., } \\
2004\end{array}$ & $\begin{array}{c}\text { Billis et al., } \\
2014\end{array}$ & $\begin{array}{c}\text { this } \\
\text { study }\end{array}$ \\
\cline { 2 - 5 } & $0.5 \mathrm{~h}$ & 652 & n.a. & 382 \\
\cline { 2 - 5 } & $2 \mathrm{~h}$ & 477 & n.a. & 458 \\
\hline \multirow{2}{*}{$\begin{array}{l}\text { down-regulated } \\
\text { mRNAs }\end{array}$} & $24 \mathrm{~h}$ & 48 & 133 & 87 \\
\cline { 2 - 5 } & $0.5 \mathrm{~h}$ & 329 & n.a. & 407 \\
\cline { 2 - 5 } & $2 \mathrm{~h}$ & 268 & n.a. & 574 \\
\hline
\end{tabular}

Table 2. Expression of proteins involved in compatible solute metabolism and transport (given are $\log _{2}$ fold changes of their levels in cells exposed for $7 \mathrm{~d}$ (proteome) or $24 \mathrm{~h}$ (transcriptome) to $684 \mathrm{mM} \mathrm{NaCl}$ versus control cells; *according to Cluster analysis 1317 shown in Fig. 2).

\begin{tabular}{|c|c|c|c|c|}
\hline Gene & Protein function & Proteome & Transcriptome & Cluster* \\
\hline sll1566 & Glucosylglycerol-phosphate synthase (GGPS) & 2.92 & 3.85 & 2 \\
\hline slr0746 & $\begin{array}{l}\text { Glucosylglycerol-phosphate phosphatase (GGP- } \\
\text { P) }\end{array}$ & 1.19 & 1.48 & 2 \\
\hline sll1085 & Glycerol-3-phosphate dehydrogenase (GlpD) & 2.55 & 0.71 & 2 \\
\hline slr1672 & Glycerol kinase (GlpK) & 2.14 & 2.71 & 2 \\
\hline slr0747 & ATP-binding subunit of GG transporter (GgtA) & 1.83 & 2.43 & 2 \\
\hline slr0529 & $\begin{array}{l}\text { Substrate-binding subunit of GG transporter } \\
\text { (GgtB) }\end{array}$ & 1.33 & 1.48 & 2 \\
\hline slr0531 & $\begin{array}{l}\text { Integral membrane protein of } G G \text { transporter } \\
\text { (GgtD) }\end{array}$ & 2.02 & 2.13 & 2 \\
\hline slr1670 & Glucosylglycerol degrading enzyme (GghA) & 1.89 & 2.95 & 2 \\
\hline sll0045 & Sucrose-phosphate synthase (Sps) & 1.22 & 0.46 & 2 \\
\hline slr0953 & Sucrose-phosphate phosphatase (Spp) & 1.04 & 1.26 & 2 \\
\hline
\end{tabular}


Table 3. Salt-regulated proteins that are involved in general stress tolerance. Given are $\log _{2}$ fold changes of their protein and corresponding mRNA levels in cells exposed for different times to $684 \mathrm{mM} \mathrm{NaCl}$ versus control cells.

\begin{tabular}{|l|l|c|c|c|c|}
\hline Gene & Protein function & Proteome & \multicolumn{3}{|c|}{ Transcriptome } \\
\hline & & $\mathbf{7 ~ d}$ & $\mathbf{2 4} \mathbf{~ h}$ & $\mathbf{2 ~ h}$ & $\mathbf{0 . 5} \mathbf{~ h}$ \\
\hline sll1863 & Unknown protein & 3.73 & 5.18 & 7.47 & 7.49 \\
\hline sll1862 & Unknown function & 3.30 & 4.00 & 5.28 & 5.30 \\
\hline sll1037 & Putative component of TRAP transporter & 3.01 & 0.63 & 0.17 & 0.38 \\
\hline \multirow{2}{*}{ slr0750 } & Light-independent protochlorophyllide reductase subunit & & & \\
\hline slr0786 & Methionine aminopeptidase B & 3.00 & 1.29 & -1.88 & -0.86 \\
\hline slr0967 & Hypothetical protein, involved in stress responses & 2.83 & 4.77 & 5.32 & 0.95 \\
\hline Sll0528 & Putative zinc metalloprotease & 2.43 & 3.00 & 3.28 & 0.27 \\
\hline sll7064 & CRISPR-Cas system 2 & 4.19 & 6.22 & 1.61 \\
\hline sll0248 & Flavodoxin (IsiB) & 2.18 & 0.57 & -2.56 & -1.00 \\
\hline sll1988 & 33 kDa chaperonin (HSP33) & 2.15 & 1.15 & 4.27 & 0.89 \\
\hline sll0947 & Light-repressed protein A, LrpA & 2.06 & 0.42 & 0.21 & 0.35 \\
\hline slr2019 & Putative ATP binding subunit of ABC transporter & 1.83 & -1.69 & -2.54 & 0.84 \\
\hline slr1894 & General stress protein MrgA/Dps & 0.94 & 1.06 & 2.09 & 1.16 \\
\hline sll1514 & 16.6 kDa small heat shock protein, molecular chaperon & -1.68 & 0.91 & 5.85 & 4.25 \\
\hline
\end{tabular}

Table 4. Salt effects on proteins involved in central carbon metabolism (given are $\log _{2}$ fold changes of their levels in cells exposed for $7 \mathrm{~d}$ (proteome) or $24 \mathrm{~h}$ (transcriptome) to 684 $\mathrm{mM} \mathrm{NaCl}$ versus control cells)

\begin{tabular}{|l|l|c|c|}
\hline Gene & Protein function & Proteome & Transcriptome \\
\hline slr0394 & Phosphoglycerate kinase & 0.76 & 0.58 \\
\hline slr0884 & Glyceraldehyde-3-phosphate dehydrogenase 1, Gap1 & 0.75 & 1.35 \\
\hline sll0842 & Neopullulanase, NpIT & 1.28 & 0.83 \\
\hline slr0237 & Glycogen debranching enzyme 1, GlgX1 & 1.16 & 1.13 \\
\hline slr1857 & Glycogen debranching enzyme 2, GlgX2 & -1.12 & -1.12 \\
\hline slr1367 & Alpha-1,4 glucan phosphorylase & 0.61 & 0.81 \\
\hline sll1356 & Glycogen phosphorylase 2, GlgP2 & 0.08 & 0.53 \\
\hline sll0587 & Pyruvate kinase 1 (PK 1) & 0.76 & 0.60 \\
\hline sll1275 & Pyruvate kinase 2 (PK 2) & -0.45 & -0.78 \\
\hline slr0301 & Phosphoenolpyruvate synthase & -1.19 & -0.97 \\
\hline
\end{tabular}

